\title{
Nuovi dati sugli edifici termali di Sabratha
}

\author{
Rosa Maria Bonacasa Carra, Nicola BonaCASA
}

\begin{abstract}
The architecture of the six Roman baths in Sabratha developed in the second century during the reorganization of the urban space after the earthquake of the Flavian period. They are all well organized in the axial arrangement of the cold and hot baths, with frigidarium as the focus of the structure, served by apodyteria, environments with benches for visitors and latrinae, and also with appropriate paths and well-wishers (the mosaics in the so-called Terme del Teatro) or marble statues (in the latrine of the so-called Terme a mare). The spaces for the hot bath stand out for the sequence of the laconicum, the tepidarium and of the calidarium, independent and equipped with the praefurnia. In the Forum area the 'Terme a mare' imposed themselves for its original technical and structural insights aimed at obtaining a building area bigger than the tectonics of the soil could then ensure. The 'Terme del Teatro' on their turn spearheaded the experimentation with new analytical techniques aiming at the restitution of the original project.
\end{abstract}

Keywords: Sabratha, Roman baths, architecture, paths, wishers

English title: New Data on the Roman Thermal Baths of Sabratha

Rosa Maria Carra Bonacasa, Centro di Ricerca per l'Archeologia del Mediterraneo (CeRAM), Università degli studi di Palermo, Palermo; rosamaria.carra@unipa.it,rosamaria.carra@libero.it

Nicola Bonacasa è scomparso improvvisamente il 30 novembre del 2015. A Lui, caro e insostituibile Compagno nel lungo e difficile percorso della vita, dedico queste pagine a testimonianza dei molti anni condivisi nelle ricerche a Sabratha.

Nell'ambito dei progetti promossi dall'Università di Palermo in Libia ${ }^{1}$, che sono stati approvati e sostenuti dai Dipartimenti alle Antichità di Tripoli e di Sabratha fin dal 1974, ci siamo occupati sistematicamente degli edifici termali di Sabratha ai fini della ricerca, del restauro e dell'edizione dei risultati ${ }^{2}$. Si presentano oggi alcuni dati di aggiornamento

\footnotetext{
${ }^{1}$ Per il tramite dell'Istituto di Archeologia, diretto da N. Bonacasa, poi Sezione Archeologica del Dipartimento di Beni Culturali, ed oggi Dipartimento Culture e Società.

${ }^{2}$ Bonacasa, Bonacasa Carra 2003; Bonacasa 2010.
} 
rispetto a quanto finora pubblicato, per dare particolare risalto alle aree occupate dagli edifici nella pianificazione della città e alle loro caratteristiche connesse con le fasi d'uso e la cronologia.

È bene precisare che nel prosieguo della nostra ricerca abbiamo avuto la convinzione che il numero delle terme presenti nella città di Sabratha al momento della sua massima espansione dovesse essere di gran lunga superiore ai sei edifici finora noti e distribuiti nelle Regiones II, III, VI e VII con una percentuale di occupazione del suolo urbano del 7,5\% su una superfice scavata pari a $115.100 \mathrm{mq}$ circa (fig. 1).

Anche se le fonti scritte ed epigrafiche non privilegiano la 'categoria delle terme', il nostro personale convincimento si è fondato su due evidenze: la generale situazione geo-topografica della città e i coefficienti climatici che connotano Sabratha. A questi si assommano due significativi dati monumentali, percepibili alle due estremità dell' area urbana indagata: ovvero nella Regio III a nord-est le c.d. 'Terme di Oceano', e nella Regio VII a sud-ovest le c.d. 'Terme dell'ufficio'. Il primo forse appartenuto ad una confraternita di marinai e commercianti di prodotti ittici finalizzati all' esportazione. Il secondo era parte integrante di una grande dimora signorile dislocata ai margini del quartiere che si sviluppò a sud-ovest, dopo la conversione in spazio urbano dell'area precedentemente occupata dai mausolei punici ${ }^{3}$. Tra questi due poli estremi si collocano gli altri quattro edifici noti:

- le grandi terme pubbliche della Regio III, preesistenti al complesso delle basiliche cristiane a nord del Teatro, il cui calidarium fu inserito nel percorso liturgico battesimale ${ }^{4}$; - le 'Terme della Regio V', a nord-ovest del Teatro;

- le terme al limite sud-est della Regio VI, nell'insula ad est della 'fontana dei Serapidi' - le c.d. 'Terme a mare' (fig. 1).

Ad eccezione delle 'Terme a mare', nella Regio II, tutti gli altri edifici risultano iscritti nello schema plano-volumetrico dell'insula di appartenenza, occupandola tutta (come avviene con le 'Terme della Regio V') oppure solo parzialmente, ma sempre bene integrati con le infrastrutture e prospicienti, almeno con due/tre lati, sulla viabilità urbana in modo da garantire il funzionamento ottimale degli impianti idrici e soprattutto di riscaldamento (fig. 1).

Queste caratteristiche si notano anche nelle 'Terme della Regio VI' (fig. 2), le cui strutture fortemente danneggiate dai terremoti del IV secolo d.C., furono riutilizzate con un poverissimo apprestamento per il bagno caldo, forse durante l'età vandalica e bizantina.

\section{'TERME DELLA REGIO VI'}

L'impianto dell'edificio (fig. 2) risale alla tarda età antoniniana e si raccorda col settore nord dell'isolato, che si sviluppa in modo indipendente ad un livello più basso, e risulta bene iscritto nello schema plano-volumetrico dell'insula. Col fronte meridionale si affaccia

\footnotetext{
${ }^{3}$ Di Vita, Procaccini, Pucci 1974-1975.

${ }^{4}$ Bonacasa Carra, Morfino 2003-2004.
} 


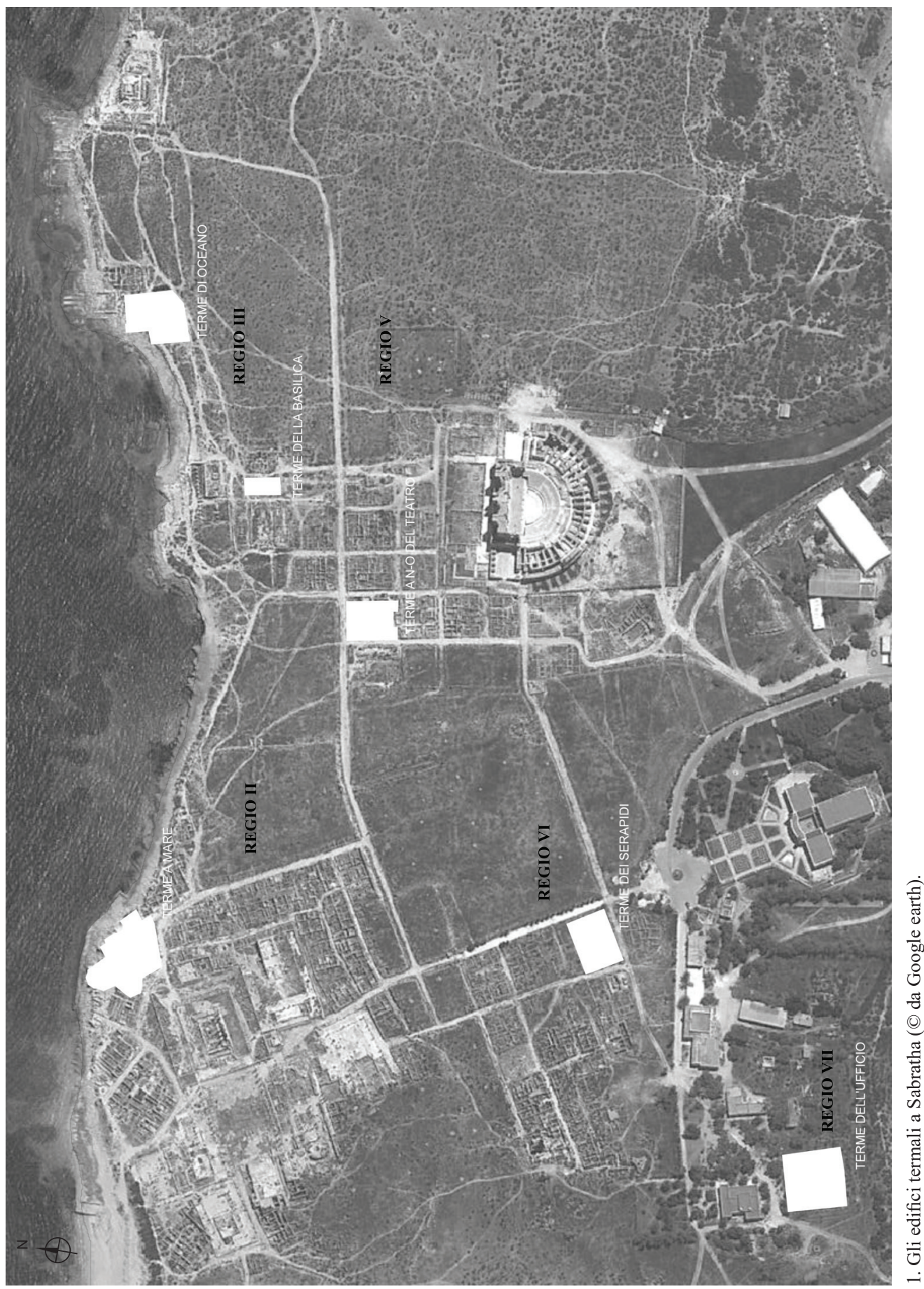




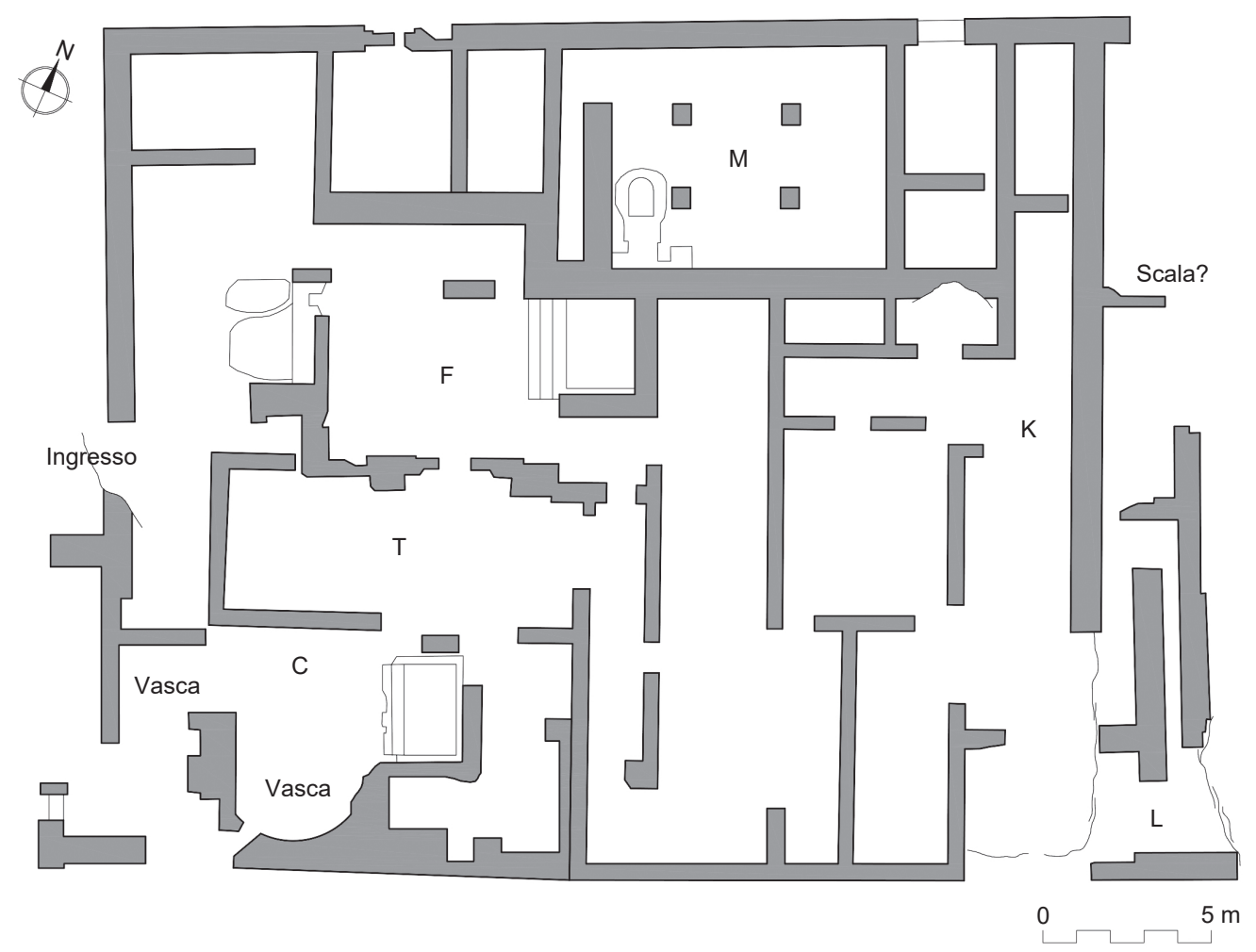

2. Pianta delle 'Terme della Regio VI', presso 'la fontana dei Serapidi' (Disegno: F. Scirè (C) Archivo CeRAM, Università di Palermo).

sul decumano e con quello ovest sul cardo, il cui tracciato si conclude nel Foro, a ridosso del portico meridionale del 'Tempio a divinità ignota' (fig. 1). Lungo il fronte orientale, sull'altro cardo, rimangono i resti appena percettibili di un canale fognario che apparteneva all'impianto di una latrina. All'estremità nord-est una rampa di gradini segnava l'ingresso di un certo prestigio - rimaneggiato nel tempo, ma mantenuto sino alla fine della vita dell'edificio - che ha sempre garantito l'accesso dal cardo ad est, superando il dislivello dei vani di servizio, per condurre il fruitore fino al piano d'uso del frigidarium (fig. 2: F).

Infatti, da un punto di vista urbanistico, gli ambienti termali erano distribuiti a sud tra il decumano ed il possente muro est-ovest che li separava dal settore nord dell'insula, dove si trovavano botteghe ed un ordinato magazzino, coperti da un tetto a terrazza sorretto da quattro robusti pilastri (fig. 2: M). All'angolo sud-ovest si trovava un pozzo che insieme alla terrazza doveva servire il grande frigidario soprastante. Quest'ultimo è stato sempre il fulcro dell'edificio per la circolazione dei bagnanti (fig. 2: F). Si trattava di un grande ambiente rettangolare, compreso tra la terrazza a nord ed il calidarium a sud. Una sola vasca, piccola ed elegante, era incassata nell'angolo nord-est della sala ed era protetta da un baldacchino che si appoggiava alle pareti e scaricava il peso su due colonne di cipollino con capitelli corinzi e basi di marmo bianco collocate agli angoli esterni della 
vasca $^{5}$. Immediatamente a sud l'ampia sala longitudinale riscaldata del tepidarium fungeva da anticamera al calidarium, che era di dimensioni più contenute, relegato all'angolo sud-ovest con tre vasche - una all'interno dell'emiciclo sul lato medirionale e due ai lati (fig. 2: T, C).

Rispetto al sistema assai ridotto dei bagni freddi, l'architettura dei bagni caldi risulta bene articolata per il numero delle vasche più volte rimaneggiate. Sicché abbiamo ipotizzato che questo impianto termale fosse al servizio della vita del quartiere. Darebbe forza a questa ipotesi anche la posizione del ninfeo di età severiana, decorato con quattro statue di Serapide, sul lato opposto del cardo ovest. Non è da escludere (anche se non è oggi possibile provarlo) che proprio la grande riserva d'acqua nella cisterna retrostante al ninfeo possa essere stata utilizzata per gli usi degli ambienti termali.

La disposizione tripartita e perfettamente assiale degli spazi destinati ai bagni è contenuta entro un percorso a linea spezzata. Superato l'ingresso con la scala a nord-est, sfruttando abilmente lo spazio disponibile, si attraversava il lungo corridoio sul lato orientale e la serie di ambienti rettangolari di servizio, per confluire in un secondo corridoio parallelo, più interno, dal quale si accedeva sia al frigidarium, sia alle due sale dei bagni caldi. Era possibile uscire dall'edificio o rifacendo lo stesso percorso retrogrado, oppure attraverso il vestibolo del frigidario, un ambiente ad L rovesciata che si affacciava sul cardo ovest.

\section{'TERME DI OCEANO'}

L'edificio situato nella Regio III (figg. 1, 3), era perfettamente inserito nell'impianto dei quartieri litoranei, lungo la via che conduceva al Tempio di Iside. Queste terme sono note per il mosaico policromo di ottima qualità, databile alla età dei Severi, che decorava il pavimento del frigidario. Si tratta di un grande emblema con la testa di una 'divinità del mare' (fig. 4), che era collocato nell'esagono centrale di un elegante tappeto geometrico (fig. 5); un ricco tralcio riempiva con foglie e racemi lo spazio dell'abside (fig. 6). Quest'ultima aveva anche un rivestimento parietale in stucco al quale apparteneva la testa di un personaggio calvo e barbato (un Sileno?) con una corona di foglie e bacche ${ }^{6}$. Degli altri apparati decorativi superstiti fanno parte le due fasce a mosaico che decoravano le vasche e una soglia, pure a mosaico, decorata con sandali, strigili ed un'ampolla per olio profumato (fig. 7) che segnava il passaggio all'angolo nord-ovest tra il settore dei bagni caldi (fig. 3: 5-8) ed il frigidario. Quest'ultimo ha rappresentato per tutto il periodo di vita dell'edificio il fulcro dell'intero impianto intorno al quale ruotavano gli altri ambienti (fig. 3: F). Oggi si legge bene la fase di età severiana che è documentata come abbiamo appena detto dallo stile degli apparati decorativi.

L'accesso all'edificio avveniva da est, attraverso due ambienti di servizio comunicanti con un'area a terrazzo, con vista sul mare, che si estendeva al di sopra di magazzini sottomessi. Il percorso molto semplice da nord-est verso sud conduceva direttamente al

\footnotetext{
${ }^{5}$ Delle colonne, basi e capitelli sopravvivono alcuni resti nell'area di scavo: Bonacasa 2010.

${ }^{6}$ Barone 1994: 102, n. 407, tav. CVII.
} 


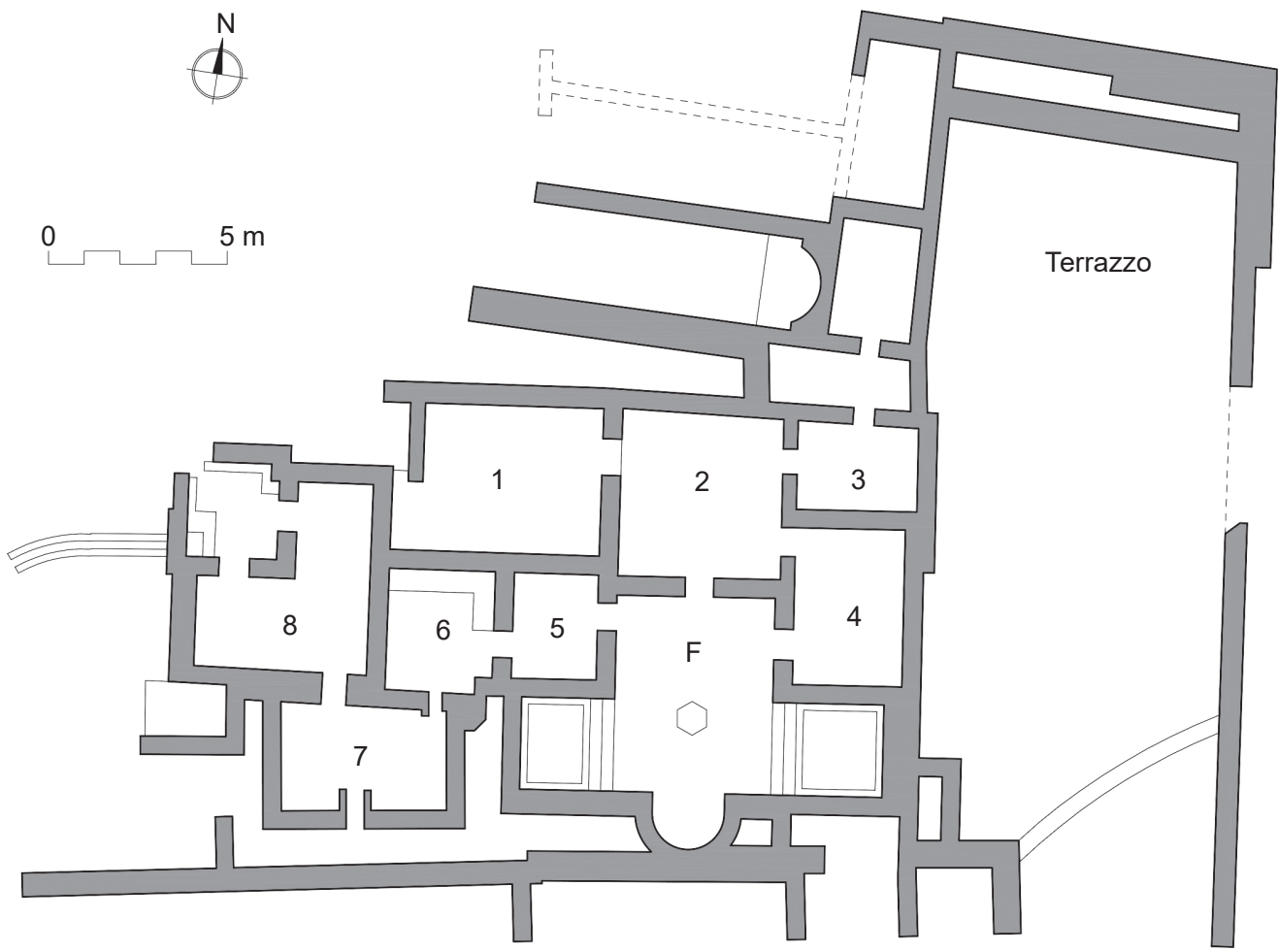

3. Pianta delle 'Terme di Oceano' (Disegno: F. Scirè $(\odot$ Archivo CeRAM, Università di Palermo).

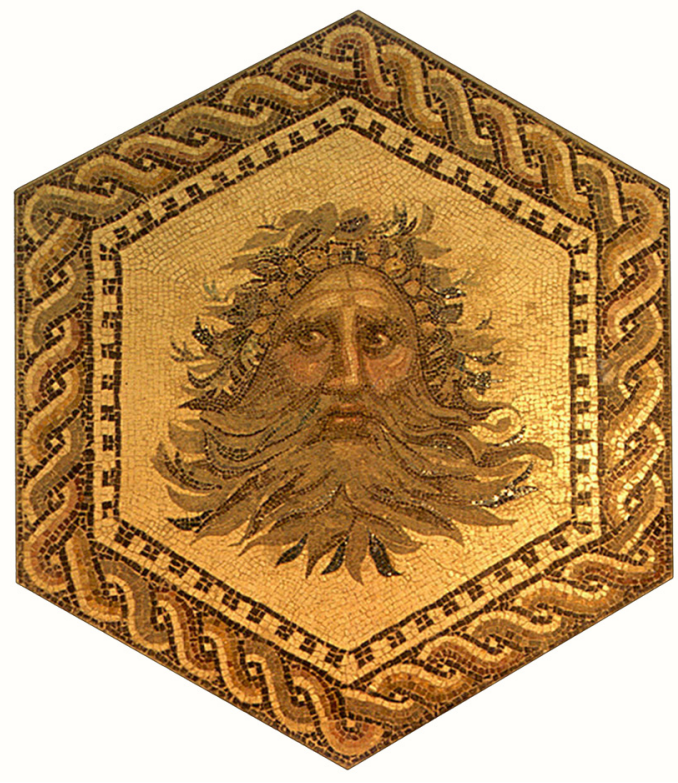

4. Mosaico con 'divinità del mare', dalle ‘Terme di Oceano' (Fot. G. Cappellani (C) Archivo CeRAM, Università di Palermo). 
frigidario attraverso gli spogliatoi e le salette di attesa (fig. 3: 1-4). Da qui si passava agli ambienti riscaldati, con i pavimenti sorretti da suspensurae e condotti di riscaldamento alle pareti (fig. 3: 5-8). Erano tutti concentrati nell'angolo sud-ovest, come nelle 'Terme della Regio VI' (fig. 2). Era possibile anche raggiungere direttamente il tepidario ed il laconico $^{7}$, per concludere il percorso di fruizione nella grande sala del frigidario. Durante i lavori di restauro ${ }^{8}$ (eseguiti proprio nel frigidarium, per procedere al distacco ed al risanamento del pavimento musivo di età severiana) è stato rinvenuto il sedile di una vasca (fig. 8), disposta in senso nord-sud e addossata ad un possente muro di conci, che era orientato est-ovest e divideva in due parti l'ambiente. Era ovvio che si trattava dei resti di un edificio termale precedente. Poiché il mosaico di età severiana era stato distaccato, abbiamo deciso di sondare la zona, con una serie di saggi mirati al riconoscimento delle fasi delle terme.

I risultati raggiunti sono riportati qui di seguito:

a) Nel corso del I secolo d.C., sicuramente nella seconda metà, è stato costruito un edificio termale di notevole ampiezza, del quale, oltre alla vasca suddetta, orientata nord-sud, restano diversi muri le cui fondazioni, tutte rasate, occupano l'estesa area a sud dell'attuale edificio severiano.

b) Tra la fine dell'età traianea e l'inizio di quella adrianéa, per un evento che ignoriamo, forse un terremoto, l'edifico è crollato. I suoi resti murari e gli intonaci dipinti sono stati riadoperati come materiali di riempimento assai compattato, e livellato da uno strato di calce.

c) Sul precedente è sorto in età adrianea un secondo edificio termale di cui conosciamo soltanto l'emblema quadrato con la testa di Diana-Luna ${ }^{9}$ (fig. 9) che era collocato al centro della sala e che fu rinvenuto sottomesso all'emblema esagonale di età severiana (fig. 5), in occasione dei restauri condotti negli anni quaranta del secolo scorso. Il mosaico pavimentale che contornava il quadro di Diana-Selene è andato perduto. Di esso abbiamo riconosciuto solo qualche traccia in alcune file di tessere bianche intercettate sotto l'impianto delle due vasche.

d) Infine, andate in disuso le strutture del II secolo d.C., probabilmente agli inizi del III, venne realizzato il piccolo e raffinato edificio, che oggi vediamo perfettamente inserito nell'impianto urbanistico del quartiere (fig. 3).

\footnotetext{
${ }^{7}$ I dati di scavo non consentono di riconoscere tra gli ambienti riscaldati un vero e proprio calidarium. Sarebbe 1'unico esempio a Sabratha! Va comunque precisato che l'abbandono definitivo del sito si colloca già agli inizi del IV secolo d.C., e che in seguito al terremoto del 365 d.C. la zona fu adoperata come cava, e frequentata saltuariamente, finché non fu occupata da un cimitero che è possibile collegare con la presenza, più ad ovest, delle Chiese 3 e 4. Vedi: Cipriano 2012.

${ }^{8}$ Negli anni 2000-2002, le campagne di restauro sono state dedicate al risanamento dei mosaici del frigidario: l'operazione di un nuovo strappo, dopo quello degli anni '40, era stata sempre rimandata sia dagli studiosi italiani sia dalle autorità libiche, a causa dei rischi connessi con il ridotto spessore delle tessere, in particolare di quelle di calcare bianco. Ma l'accurato e lento lavoro di sezionamento e strappo del mosaico effettuato dai restauratori della Missione dell'Università di Palermo ha garantito la totale immunità dell'opera. Bonacasa 2004.

${ }^{9}$ Bonacasa 2004.
} 


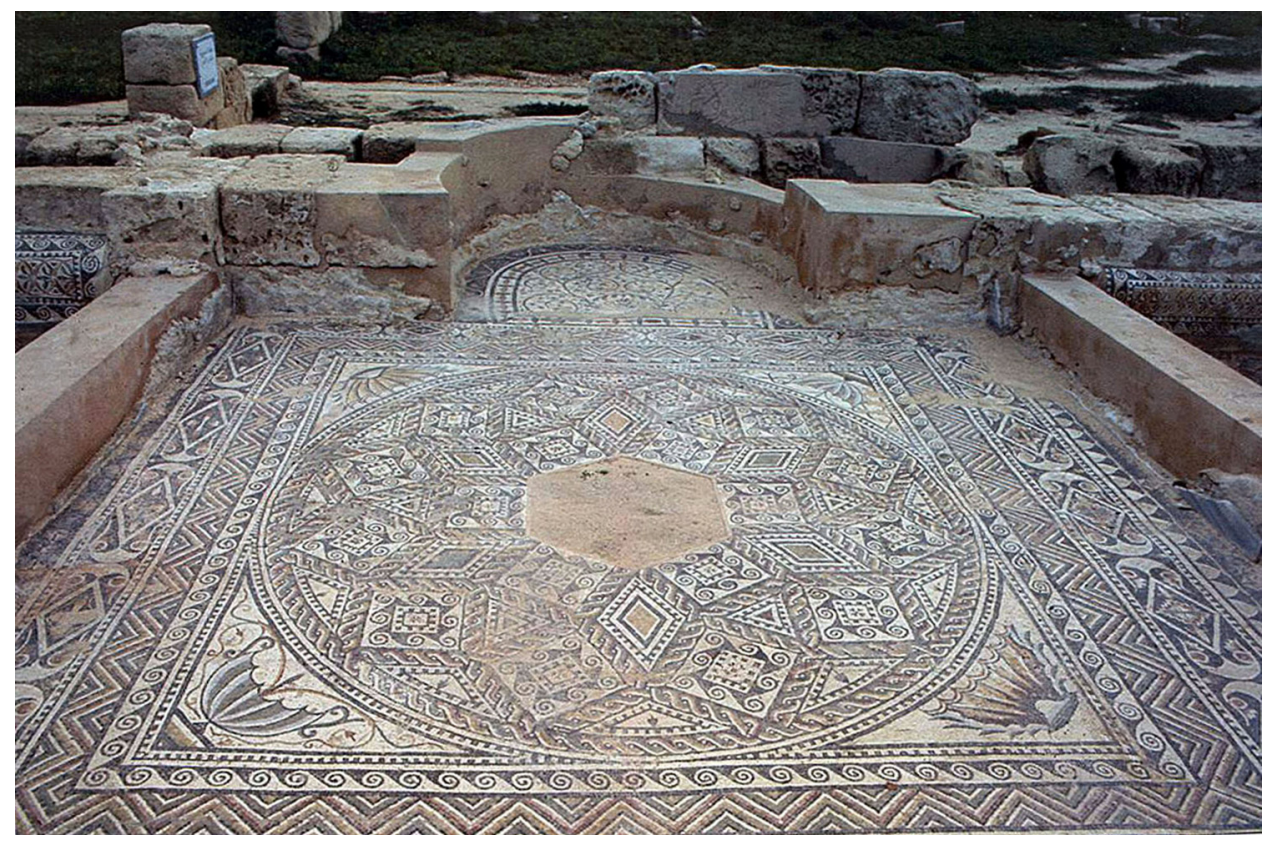

5. 'Terme di Oceano': il pavimento a mosaico della sala del frigidarium (Fot. N. Bonacasa @ Archivo CeRAM, Università di Palermo).

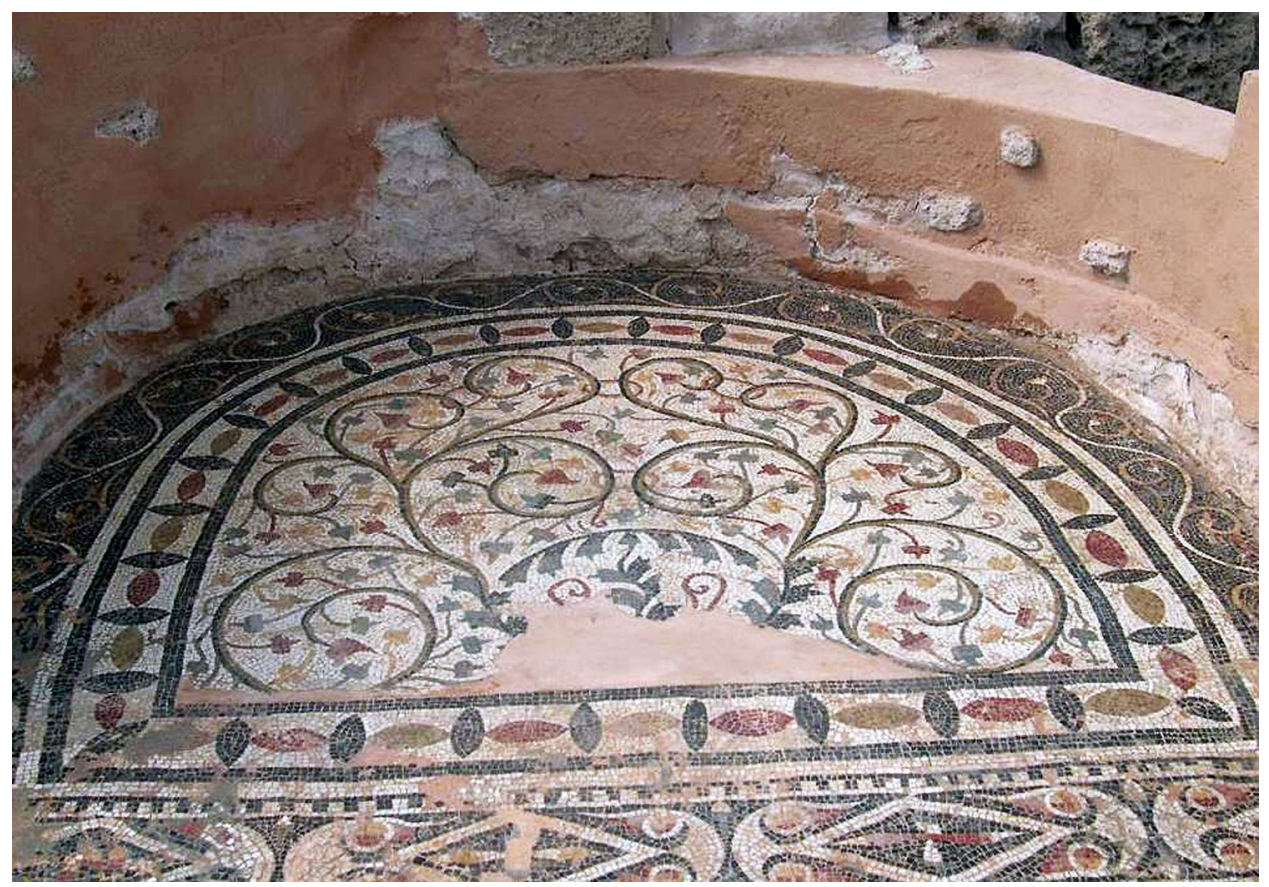

6. 'Terme di Oceano': il mosaico nell'abside del frigidarium (Fot. N. Bonacasa C Archivo CeRAM, Università di Palermo). 


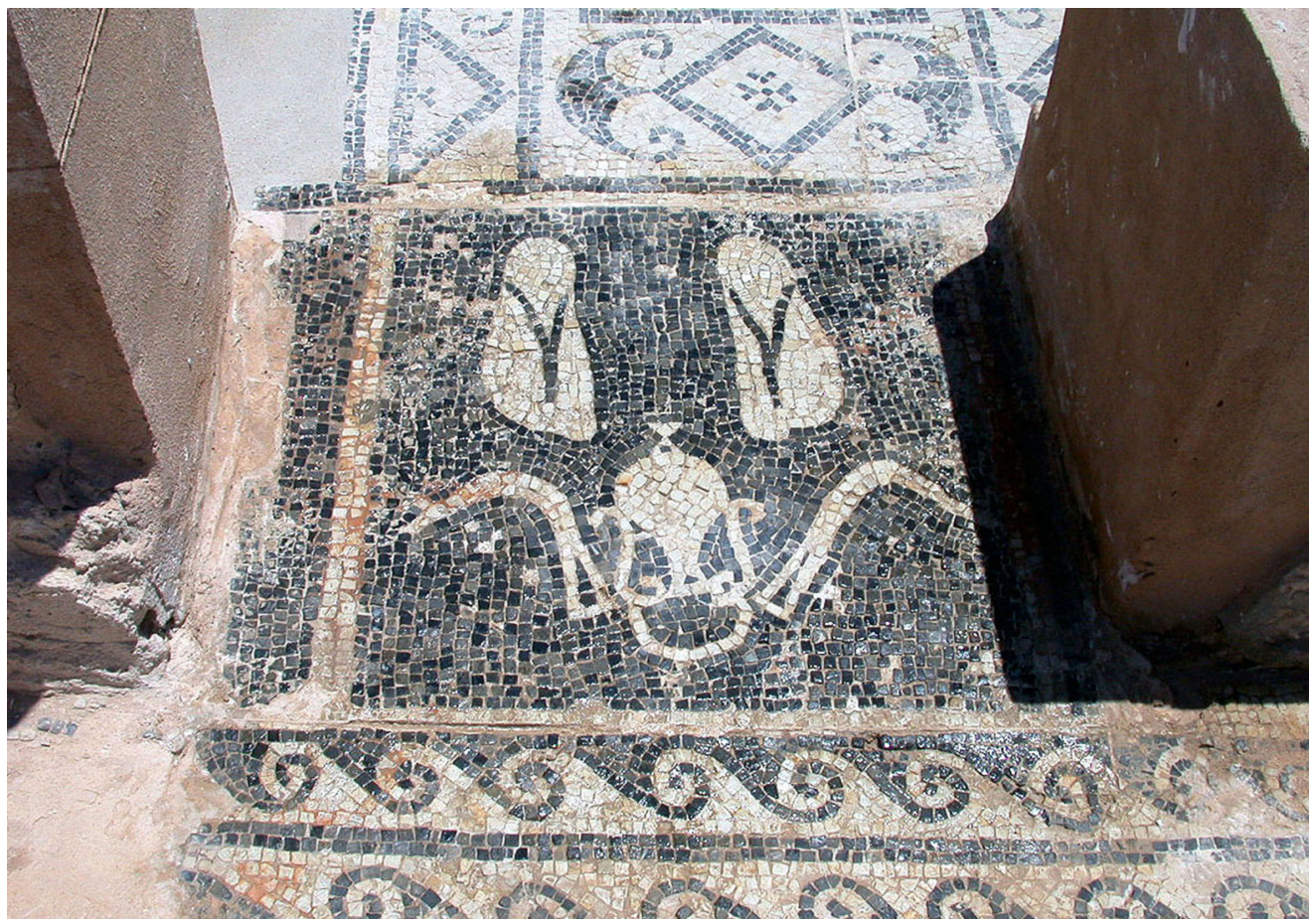

7. 'Terme di Oceano': la soglia tra il frigidario e i bagni caldi (Fot. N. Bonacasa $\subset$ Archivo CeRAM, Università di Palermo).

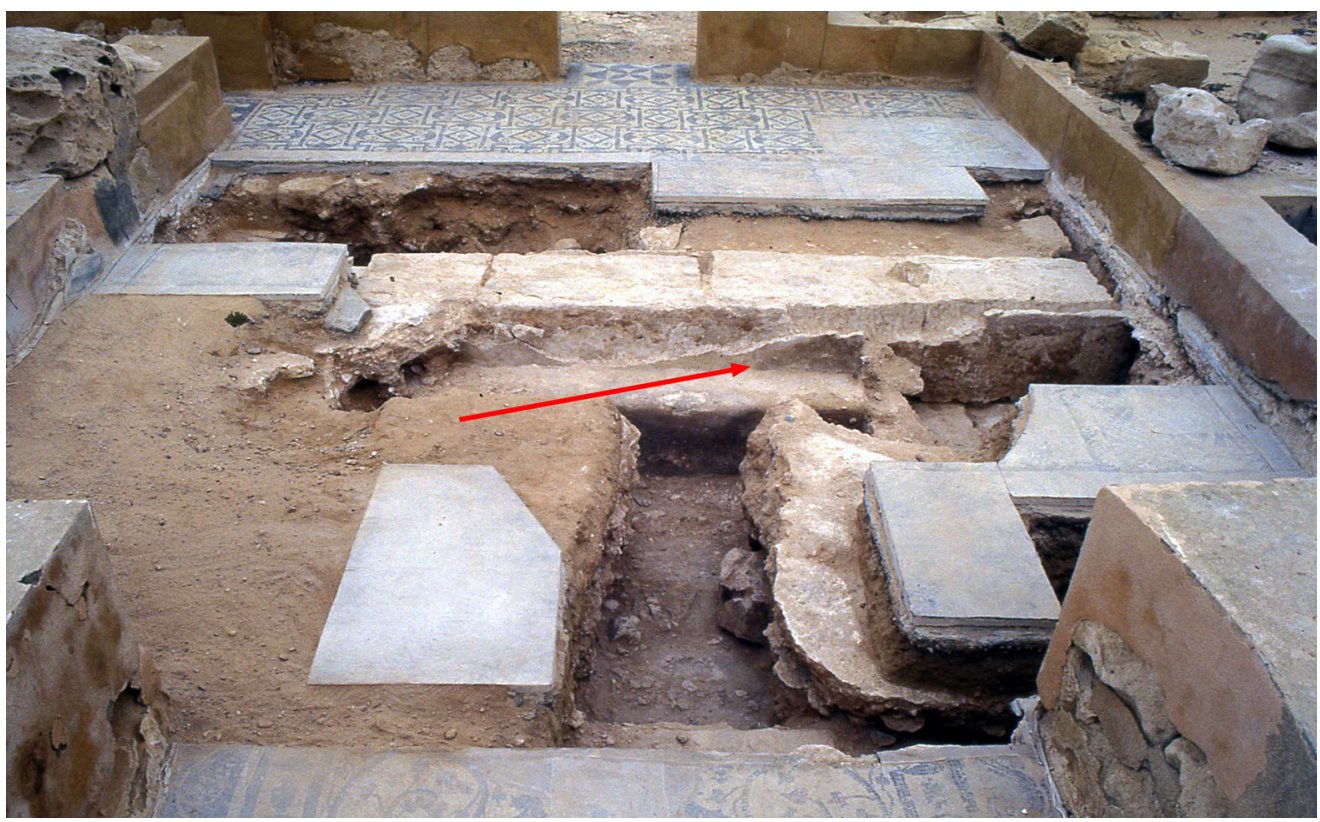

8. 'Terme di Oceano': la vasca del primitivo frigidario (Fot. N. Bonacasa (C) Archivo CeRAM, Università di Palermo). 


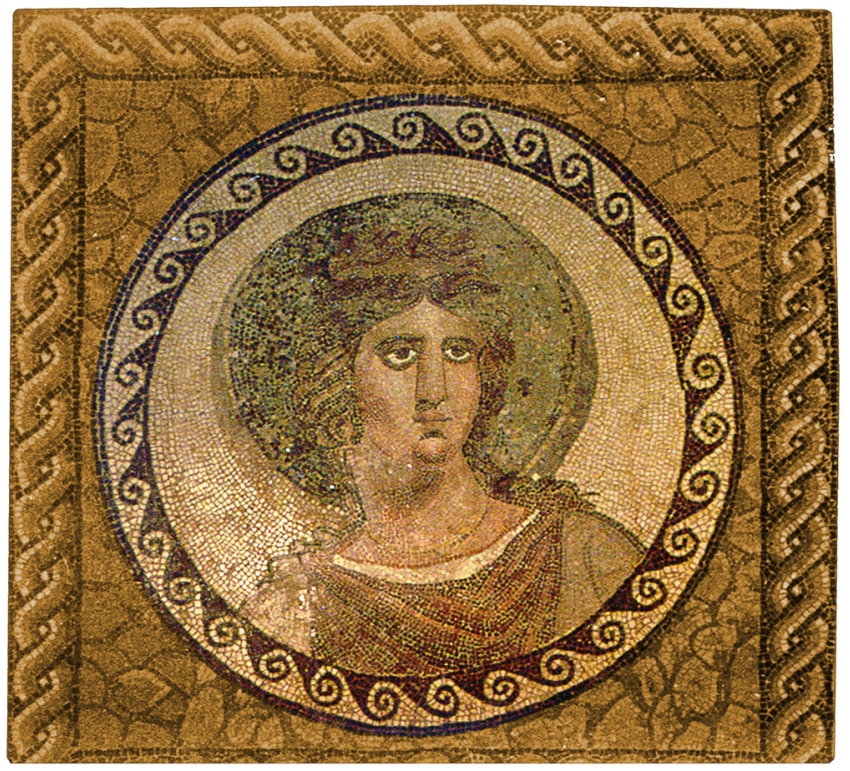

9. 'Terme di Oceano': mosaico con la testa di Diana/Luna (Fot. G. Cappellani (C) Archivo CeRAM, Università di Palermo).

\section{'TERME DELLA REGIO VII'}

Questo edificio ${ }^{10}$ (fig. 10), come le due terme precedenti, presenta una pianta organica abbastanza contenuta, con uno sviluppo ovest-est per gli ambienti dei bagni e nord-sud per gli accessi. Le sale di intrattenimento e le latrine (fig. 10: A, L) si affacciavano su un portico colonnato lungo la fronte ovest. La realizzazione del progetto - secondo i dati emersi dai saggi di scavo condotti soprattutto nel portico - si data tra la seconda metà del II secolo d.C. ed i primi anni del III. Le strutture sono rimaste sostanzialmente invariate fino alla distruzione avvenuta col terremoto del 365 d.C. Pochi interventi hanno riguardato nel tempo la funzionalità di alcuni ambienti di servizio, ad esempio le latrine, i depositi d'acqua ecc., nonché il rifacimento di alcuni pavimenti a mosaico, specialmente quello del frigidario che viene datato dagli editori entro il primo venticinquennio del IV secolo d.C.

Interessante e certamente canonico è il percorso di fruizione che partiva sicuramente dall'estremità nord-ovest con un vestibolo che introduceva direttamente all'ampia sala rettangolare del frigidario con due belle vasche, esagonale quella nord e absidata quella sud (fig. 10: F). Immediatamente ad est si sviluppava il settore dei bagni caldi preceduto da due tepidari (fig. 10: T1, T2) con sedili addossati sui lati brevi entro nicchie semicircolari e con stretti passaggi di comunicazione col frigidario. Lo sdoppiamento dei tepidari agevolava la circolazione dei bagnanti a senso unico. Partendo dal tepidario più grande a sud-ovest si sostava nell'ampio vano absidato dotato di panchine sui lati lunghi, che si ritiene fosse un destrictarium (fig. 10: D), quindi transitando per il piccolo laconicum intermedio (fig. 10: Lac)

\footnotetext{
${ }^{10}$ Indagate e pubblicate da Brecciaroli Taborelli 1974-1975: 113-146.
} 


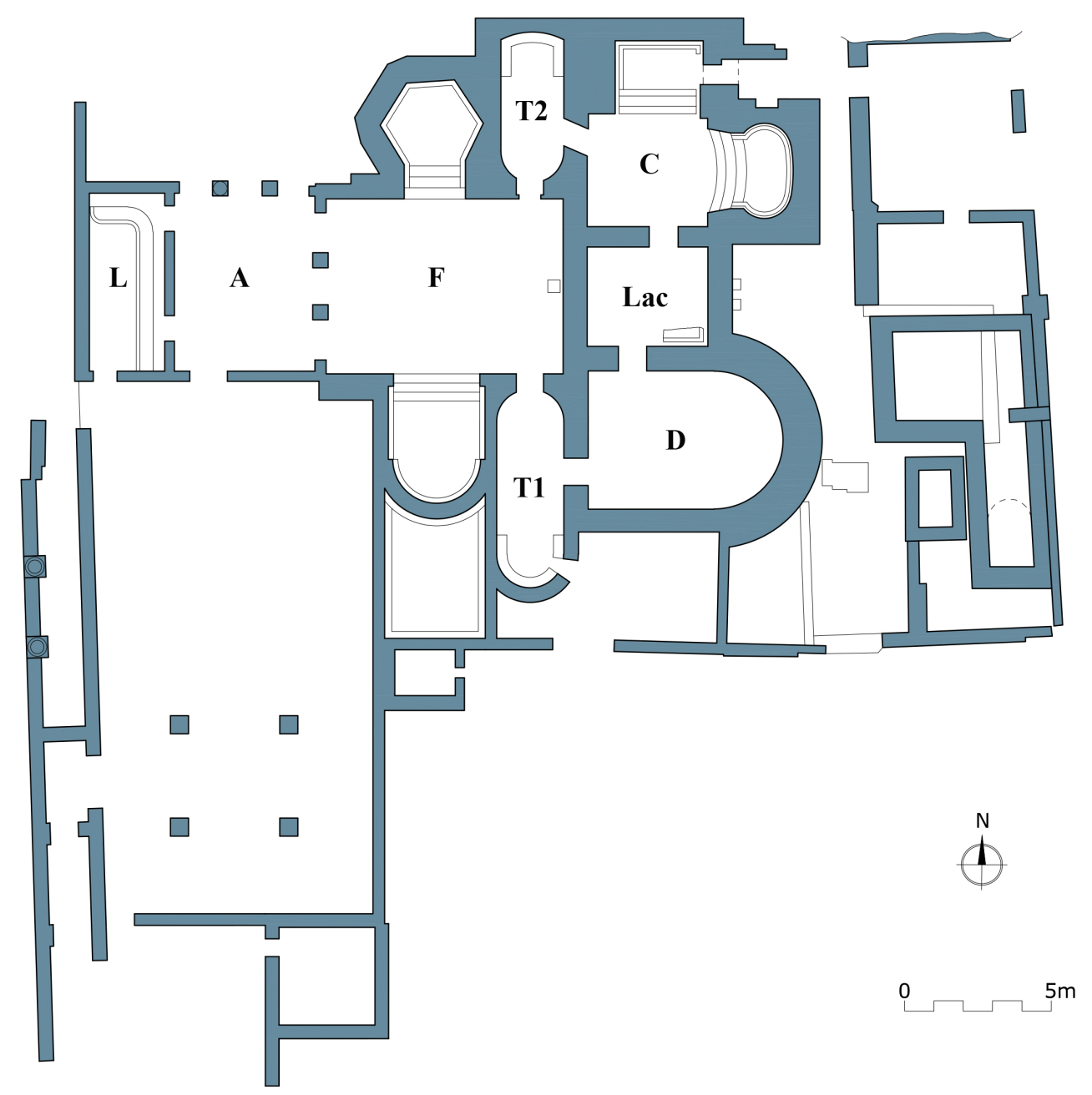

10. Pianta delle 'Terme della Regio VII' (Disegno: F. Scirè $(C)$ Archivo CeRAM, Università di Palermo).

si guadagnava la sala del calidarium con due belle vasche, rettangolare quella nord e ovoidale quella est (fig. 10: C); infine si transitava per una breve sosta nel secondo tepidario, a nord-ovest (fig. 10: T2), prima di accedere nuovamente alla sala del frigidario. Questo percorso di fruizione rimarrà invariato anche nella seconda fase - quando il vano di servizio a sud del destrictarium viene trasformato in un nuovo tepidario, e vengono mantenenuti in funzione anche gli altri due.

\section{TERME 'A NORD-OVEST DEL TEATRO’}

Occupavano l'insula 1 della Regio V. Negli anni 2001-2003, 2004-2005 e 2013, sono state oggetto di interventi di restauro e di risanamento dei bagni caldi a nord e di estesi, 


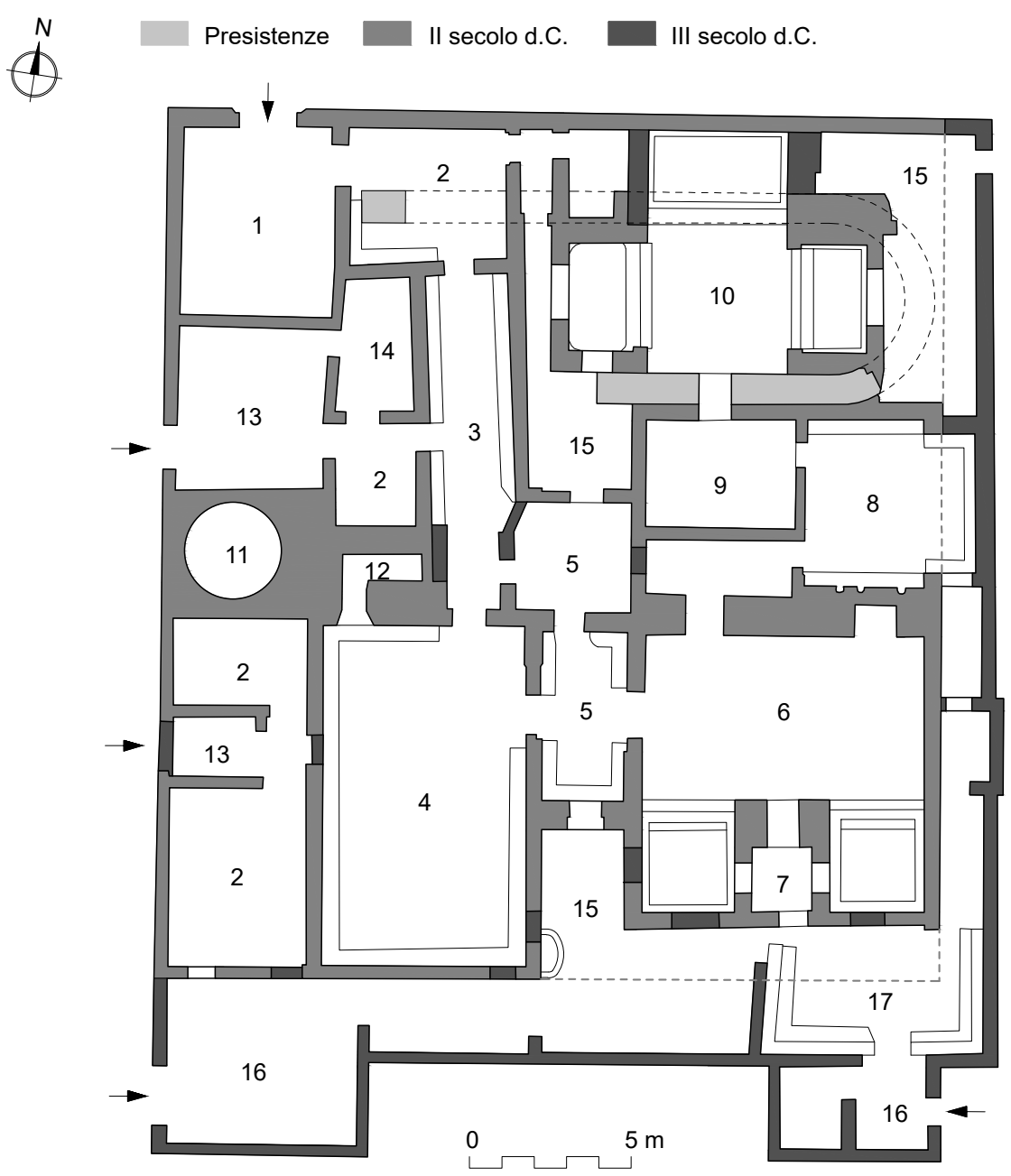

11. Pianta delle 'Terme della Regio V': 1. ingresso dal decumano; 2. spogliatoio; 3. corridoio; 4. salone; 5. anticamera; 6. frigidarium; 7. pozzo luce; 8. tepidarium; 9. laconicum; 10. calidarium; 11. pozzo; 12. piattaforma di sollevamento dell'acqua; 13. ingresso dal cardo; 14. ambiente di servizio; 15. ambiente di servizio scoperto; 16. ingresso latrine; 17. latrine (Disegno: F. Scirè (C) Archivo CeRAM, Università di Palermo).

impegnativi lavori di restauro ai mosaici del corridoio di accesso e del frigidario, durante i quali abbiamo svolto anche alcuni saggi di scavo che sono stati utili per determinarne le fasi ${ }^{11}$ (fig. 11).

Si tratta dell'edificio termale più prestigioso fra quelli presenti nel Quartiere del Teatro, che ebbe una pianificazione urbanistica, con l'ampliamento verso est-nord-est del primitivo tessuto - quando Sabratha, divenuta colonia sotto Antonino Pio, nella seconda metà del

\footnotetext{
${ }^{11}$ Bonacasa Carra 2012; Bonacasa Carra, Scirè 2012.
} 
II secolo d.C. godette di un periodo di particolare prosperità. Con la ripetizione di insulae quadrangolari e parallele, orientate verso il mare, che si attestavano sugli assi stradali nord-sud perpendicolari alla linea di costa ed inglobavano il decumano principale e quelli minori, abbreviando le percorrenze, con un migliore rapporto distanza-funzionalità, in questo nuovo quartiere sembra avere prevalenza l'edilizia privata, per uso sia abitativo che commerciale.

Eppure le Regiones III-V che rientravano nel nuovo assetto della città spiccano anche per la presenza di un tempio dedicato ad Ercole ${ }^{12}$, di un altro a Iside ${ }^{13}$ e di ben tre edifici termali pubblici, pressoché coevi nell'impianto, che tuttavia ebbero vicende assolutamente diverse (fig. 1).

Le 'Terme della Regio V' si distinguono per l'apprezzabile stato di conservazione delle strutture, cosa abbastanza insolita a Sabratha (figg. 11-12). Questa particolare caratteristica ci ha consentito di sviluppare un'adeguata analisi strutturale e di verificare l'ipotesi di progetto per una restituzione tridimensionale (fig. 12) con l'impiego di una unità di misura ' $\mathrm{M}$ ' (= 10 x 10 p.r.), che abbiamo già sperimentato nello studio delle basiliche cristiane ed applicato anche a quello delle 'Terme della Regio II'14. Le strutture sono riconducibili a due periodi distinti, il secondo dei quali si articola in due fasi (fig. 4). Il periodo I riguarda le preesistenze del calidarium, collocabili tra il I secolo a.C. ed il I d.C. secondo i dati emersi dai pochi materiali ceramici rinvenuti nelle stratigrafie. Tali preesistenze consistono in un grande ambiente quadrangolare, in origine absidato ad est, di cui sono sopravvissuti due possenti muri: quello settentrionale - orientato est-ovest e successivamente rasato per consentire la proiezione del nuovo impianto termale sul decumano - e quello meridionale, riutilizzato per separare il calidarium dagli altri ambienti riscaldati.

Infatti l'edificio termale che oggi è possibile leggere nelle strutture e nella sua pianificazione progettuale nel corso del II secolo d.C. occupa un intero isolato di 8 x $9 \mathrm{M}$ che si affacciava sul decumano massimo, immediatamente ad est del 'Tempio di Ercole' ed a nord di una 'Casa con Peristilio', dalla quale era separato da un decumano minore ${ }^{15}$ (figg. 1, 4).

Le terme risultano suddivise in due settori ben distinti: quello dei bagni che occupa la metà orientale dell'insula e quello dei servizi collocati nell'altra metà con un vestibolo a nord-ovest, le sale di transito e di attesa, un ampio salone (fig. 11: 1-4), forse un ginnasio coperto (o palestra?). Spicca una cisterna circolare (n. 11), dotata di una piattaforma per la macchina di sollevamento dell'acqua (n. 12), che era connessa agli ambienti adiacenti sul fronte ovest, due dei quali (n. 13) con ingresso direttamente dal cardo.

Come altri edifici termali nord-africani le 'Terme della Regio V' non sfuggono alle leggi romane dell'economia della pianta, della razionalità degli spazi, della simmetria degli ambienti iterata come bilancia di equilibri ${ }^{16}$. Ben distinto risulta, infatti, il settore dei bagni caldi, a nord da quello dei bagni freddi, a sud.

${ }^{12}$ Caputo, Ghedini 1984; Aiosa 2012; Aiosa 2013: 193-202.

${ }^{13}$ Pesce 1953.

${ }^{14}$ Bonacasa Carra 2016.

${ }^{15}$ Aiosa 2012; Mandruzzato 2012.

${ }^{16}$ Stucchi 1957: 177-178, 184-186; Pasquinucci 1987: 72-76; Nielsen 1990: 87-90 (vol. I); Thébert 1991: 139-149; 2003: 288-318; Yegül 1992: 128-183, 186-217; Bonacasa 2010: 50. 


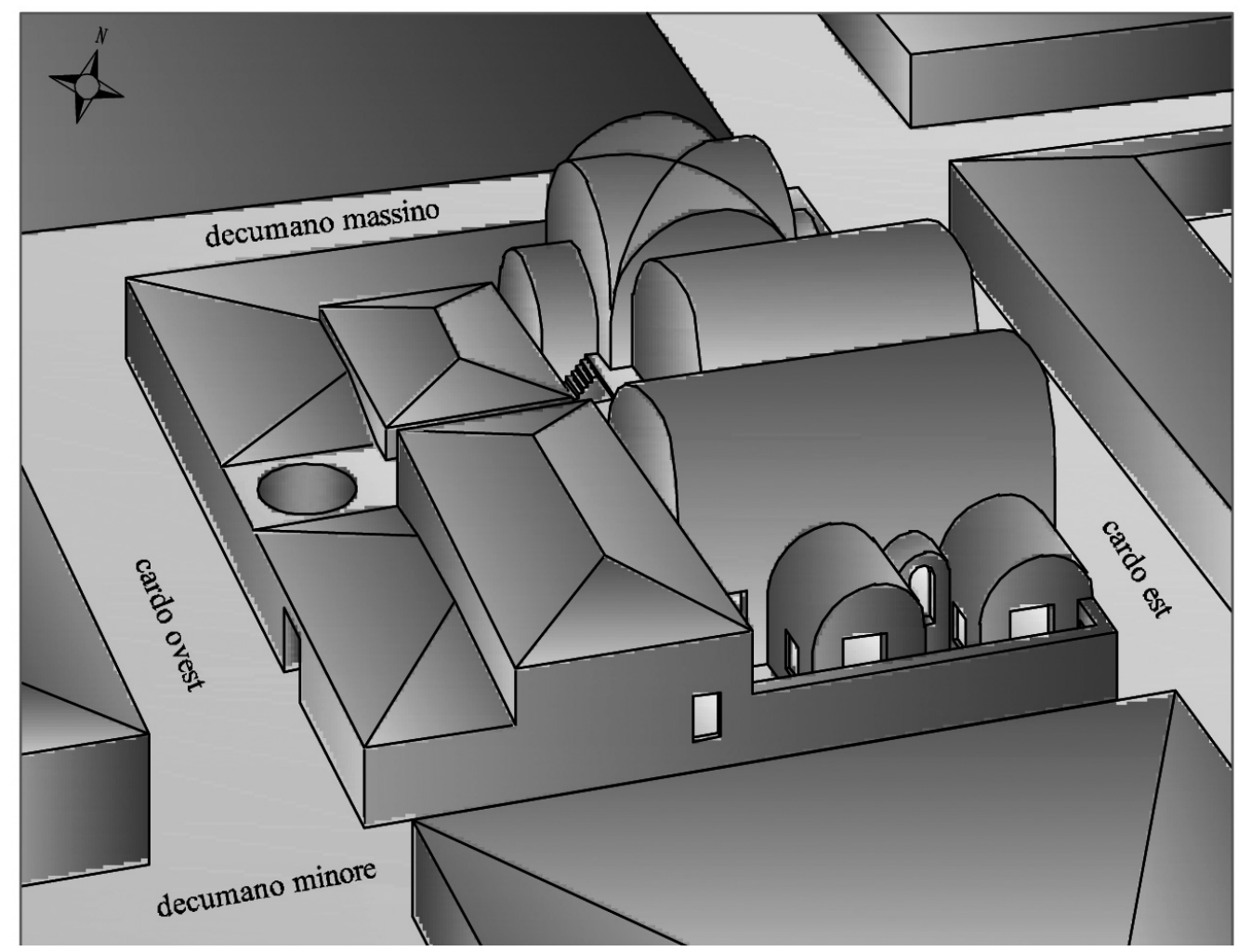

12. Ipotesi di restituzione dei volumi nelle 'Terme della Regio V' (Disegno: F. Scirè C Archivo CeRAM, Università di Palermo).

Il calidarium, compresi i corridoi di servizio, si inserisce in un rettangolo di 4 x $3 \mathrm{M}$ e occupa la metà orientale del fronte nord dell'isolato, chiuso da un muro continuo che aveva in facciata un marciapiede (fig. 12). Era una grande sala, con rivestimenti marmorei alle pareti, e pavimento a mosaico geometrico bianco e nero su suspensurae, coperta da una volta a crociera sorretta da quattro pilastri, che sviluppava un'altezza pari alla larghezza (=2 M). Due vasche gemelle contrapposte, sui lati ovest ed est, erano contenute da strutture che si addossavano al muro meridionale dell'edificio preesistente. Queste vasche erano riscaldate ciascuna da un forno autonomo, posizionato a nord, in due servizi a cielo aperto (fig. 11: 10, 15). Ciascuna vasca aveva una volta a botte, indipendente e ribassata rispetto alla crociera; entrambe si inserivano correttamente nel volume quadrangolare centrale (fig. 12) ed erano rivestite da uno spesso strato di cocciopesto, dotate di suspensurae e riscaldate alle pareti con tubuli a sezione quadrangolare.

Il laconicum a pianta rettangolare $(3 \times 2 \mathrm{M})$, coperto da una volta a botte, era ripartito da tramezzi in tre vani (nn. 8-10), di cui solo quello vicino al passaggio ad arco verso il calidarium era dotato di suspensurae e riscaldato da un forno che era alimentato dal servizio a ovest. Grossi tubuli cilindrici alle pareti, in corrispondenza di una panchina addossata al muro sud convogliavano il calore prodotto dal forno anche nel vano adiacente (fig. 11: 9). 
Un passaggio voltato ricavato nello spessore del muro meridionale immetteva nel settore dei bagni freddi.

Il frigidarium era un'aula rettangolare di dimensioni uguali al tepidarium $(2 \times 3 \mathrm{M})$ e suddivisa da un tramezzo in due parti distinte: uno spazio tripartito, per l'attesa (n. 5), e la sala con due vasche quadrangolari (n. 6), assolutamente indipendenti dalle strutture dell'aula. Mentre quest'ultima sviluppava, con la grande volta a botte decorata da stucchi, un'altezza pari alla larghezza di $2 \mathrm{M}$, ciascuna delle vasche - che affiancavano un lucernario (n. 7), con esse modulare - raggiungeva con la piccola volta a botte l'altezza massima di 1,5 M dal pavimento della sala ed era sottomessa rispetto a quest'ultimo di 0,5 M (fig. 13). La parte meridionale dell'edificio era interessata da un corridoio di servizio prospiciente sul decumano minore.

Ingresso. I fruitori potevano accedere alle sale dei bagni da tre ingressi: due sul cardo ovest ed uno a nord-ovest sul decumano massimo; altri due ingressi di servizio per i bagni caldi e freddi si aprivano sul cardo orientale (fig. 11). Il percorso dei bagnanti era sempre retrogrado a doppio senso: se entravano dal vestibolo a nord-ovest sul decumano, attraverso l'apodyterium (n. 2) e il lungo corridoio (n. 3) guadagnavano subito il disimpegno (n. 5) e gli ambienti riscaldati del laconicum (nn. 8-10), dove sostavano in attesa di accedere al bagno caldo; si trasferivano quindi nel frigidarium (n. 6) e ad operazioni concluse uscivano sul cardo ovest dopo avere attraversato il salone (n. 4) e il piccolo vestibolo (n. 16). Qualora i bagnati scegliessero il percorso più lungo: prima sostavano nel salone-palestra (n. 4) e attraverso il frigidarium accedevano alle sale riscaldate ed alle vasche calde - quindi con percorso inverso guadagnavano le uscite sul cardo ovest.

Nel Periodo II fase 2, che si può far risalire all'età dei Severi, l'edifico riceverà delle trasformazioni e piccole migliorie utili ad una più partecipata fruizione degli spazi, senza tuttavia compromettere (ne siamo sicuri!) il sistema organico delle coperture che si fondava sulla successione tra la crociera del calidarium e le due volte a botte parallele e di uguale altezza del laconicum e frigidarium per concludersi a sud con le piccole botti delle vasche fredde, perpendicolari a quella del frigidarium (fig. 12). Un tetto a capriate, più basso delle volte, copriva gli spazi di ingresso, di attesa e il salone (n. 4); un tetto ad un falda concludeva il sistema sul perimetro settentrionale ed occidentale, interrompendosi in corrispondenza della grande cisterna circolare con la piattaforma annessa.

In questa seconda fase il decumano a sud verrà invaso da una modesta latrina e un cortile con fontanella, funzionali sia alle terme che alla confinante 'Casa con Peristilio' ${ }^{17}$. I due edifici (casa e terme) costituiranno così un unico isolato sovrasviluppato in senso nord-sud, che ricalcava le dimensioni dell'insula 7, parallela ad ovest, dove tra la fine del II-inizi III secolo d.C. fu costruito il 'Tempio di Ercole' ${ }^{18}$. Anche il cardo orientale risentirà di una parziale occupazione per rimodellare gli spazi di fruizione. Nel laconicum (n. 9) fu realizzata un'esedra rettangolare con una panchina ad U rivestita di marmi. Nel calidarium l'inse-

\footnotetext{
${ }^{17}$ Vedi: Mandruzzato 2012.

${ }^{18}$ Caputo, Ghedini 1984: 10; Aiosa 2012; 2013.
} 

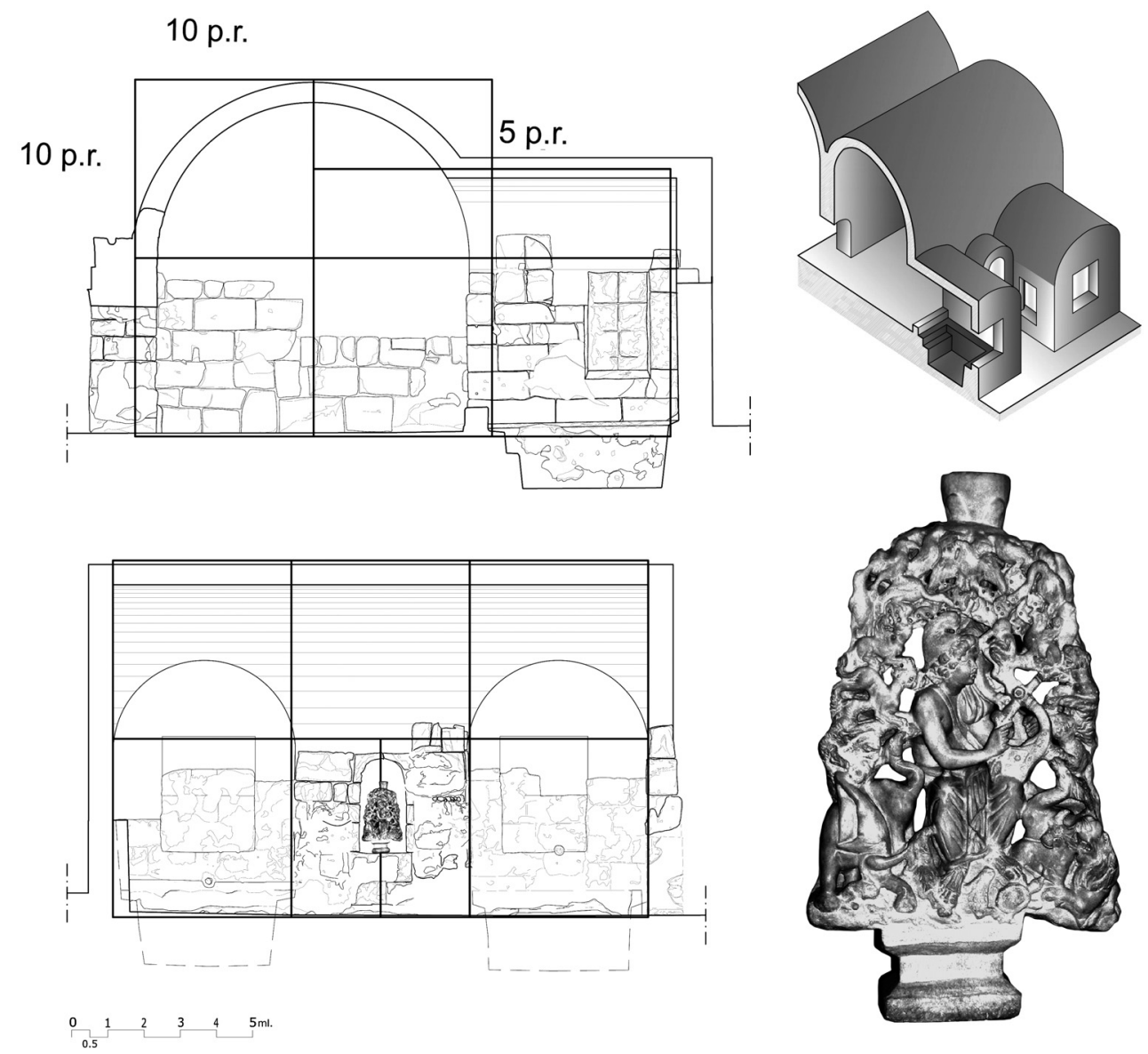

13. 'Terme della Regio V': la nicchia - lucernario tra le vasche del frigidarium e il candelabro di marmo con Orfeo cantore (Disegno: F. Scirè; Fot. G. Cappellani C Archivo CeRAM, Università di Palermo).

rimento della terza vasca a nord ha determinato il riassetto del servizio a nord-est (n. 15) con la creazione di un altro forno. Ancora qualche precisazione su altri piccoli interventi nel frigidarium, primo fra tutti la trasformazione del lucernario in una nicchia rivestita di marmi per accogliere uno splendido candelabro di marmo proconnesio, lavorato a traforo con la figura di Orfeo cantore tra gli animali (fig. 13). Il primitivo pavimento a mosaico bianco e nero è stato mantenuto solo al centro della sala con otto pannelli che fanno corona ad un largo chiusino di marmo. Nel resto della sala il pavimento è stato modificato in un semplice tessellato di cubelli di marmo bianco/grigio, interrotto davanti alle vasche da due emblemata policromi con le scritte beneauguranti SALVOM LAVISSE e BENE LABA e le immagini degli attrezzi utili al bagno (fig. 14), secondo una moda già sperimentata a Sabratha nelle 'Terme di Oceano' e come si riscontra anche a Timgad ed a Setif ${ }^{19}$.

\footnotetext{
${ }^{19}$ Warot 1960: 167-171.
} 

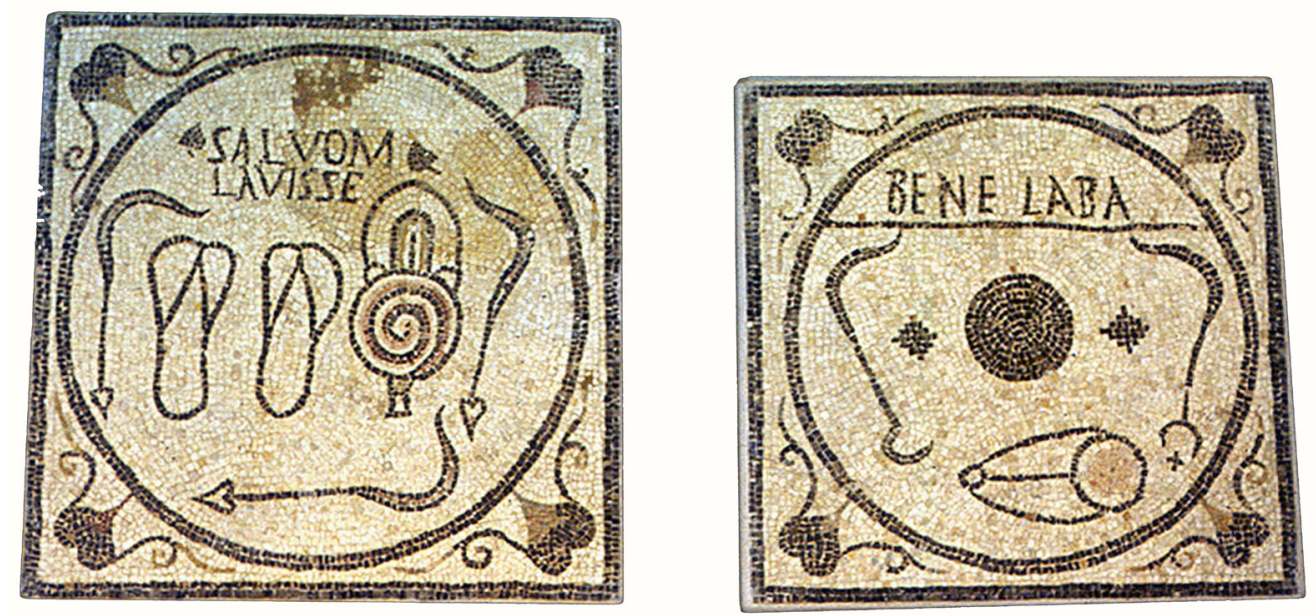

14. Mosaici del frigidarium nelle 'Terme della Regio V' (Fot. G. Cappellani (C) Archivo CeRAM, Università di Palermo).

L'aggiunta di nuovi servizi, ha conferito alle 'Terme della Regio $\mathrm{V}$ ' forme più complesse e perfino inusuali, a scapito dell' organicità che aveva contraddistinto la pianta nella prima fase. Viene cambiata in parte la funzione di alcuni spazi di transito e di sosta - senza tuttavia modificare le funzioni primarie delle sale destinate ai bagni, né il percorso retrogrado a doppio senso dei bagnati ${ }^{20}$.

Quanto alla cronologia disponiamo di maggiori dati per la fase 2, a cominciare dal candelabro con Orfeo (fig. 13) che insieme ai due mosaici policromi (fig. 14) arreda la nuova sistemazione del frigidarium ${ }^{21}$. Vanno anche ricordati altri oggetti d'uso segnalati nell'inventario di scavo $^{22}$. A questi va aggiunto un 'tesoretto' di denari di età severiana ${ }^{23}$, degli anni immediatamente successivi all'emissione di un denario di Caracalla del 205-206 d.C., che è la data più recente all'interno delle 56 monete comprese nel gruzzolo.

Gli effetti disastrosi del terremoto del 365 d.C. ${ }^{24}$ compromisero per sempre i volumi della costruzione, ma le strutture del bagno caldo continuarono, comunque, ad essere utilizzate con pochi e poveri interventi di ripristino fino all'età bizantina.

Ci resta da dire ancora di due importanti impianti a carattere pubblico presenti a Sabratha: le 'Terme sotto la Basilica 3' e le 'Terme a mare'.

${ }^{20}$ Bonacasa Carra 2016.

${ }^{21}$ Floriani Squarciapino 1941; Bonacasa 1960.

${ }^{22}$ Come le lucerne (con una preponderanza di quelle di III secolo d.C. anche se non mancano le tripolitane di IV, gli aghi crinali, il pendente di madreperla a forma di foglia ed i monili d'oro le due statuette quella di Iside, di avorio e di Mercurio in bronzo, il bruciaprofumi con testa di Serapide, il cucchiaio e la tessera d'osso: Bonacasa Carra 2016.

${ }^{23}$ Macaluso 1995: 75-81.

${ }^{24}$ Di Vita 1980: 303-307; 1990: 425-496; Bonacasa, Bonacasa Carra 2003: 403-404, 407, 412, 417-418. 


\section{'TERME SOTTO LA BASILICA 3'}

Di questo edificio nella Regio III, a nord del decumano massimo, si conservano solo le strutture dei bagni caldi e le riserve d'acqua. Infatti l'insula 8 su cui insisteva fu riutilizzata dopo il terremoto del 21 luglio del 365 d.C. per la realizzazione del complesso episcopale paleocristiano $^{25}$. Il calidarium è l'unica parte delle terme che rimase perfettamente funzionante per essere inserito nel percorso liturgico battesimale.

Già il Lassus a proposito dei gruppi episcopali di Djemila, Tipasa, Timgad, Sbeitla 2, Uppenna, Mactar ed Ippona segnalava l'importanza della presenza di un impianto termale vicino al battistero, come indizio di un avanzato stadio di sviluppo del gruppo episcopale. Lo studioso ipotizzava, inoltre, che l'utilizzo dei bagni caldi termali nel percorso della liturgia battesimale, fosse dettato dalla necessità di atti purificatori precedenti il battesimo, come ci insegnano Tertulliano e S. Agostino ${ }^{26}$.

Le 'Terme sotto la Basilica 3' (fig. 15) erano un impianto pubblico di notevole imponenza, che per oltre metà è andato perduto. Ma se si guarda all'estensione totale ipotizzabile in m 50 × 60 circa ed alla pianificazione antoniniana della zona orientale della città, non v'è dubbio che l'edificio in origine dovette essere il primo e il vero impianto termale al servizio del porto e del teatro ${ }^{27}$. I pochi resti dei bagni caldi (conservati su un'area di m 32,3 x 19,5), consistono in un calidarium con tre vasche inserite in altrettante nicchie, quella rettangolare nella parete opposta all'ingresso e le altre due semicircolari contrapposte sui lati brevi (fig. 15: C). Due ampi vani di forma ellittica, contigui e paralleli, introducevano, attraverso passaggi a sguincio, alla sala con le vasche; all'origine erano anche essi riscaldati (fig. 15: L). Alle spalle del calidarium, immediatamente ad est si trovano i servizi per il riscaldamento, ed una grande cisterna a pianta rettangolare, sotterranea, con una piattaforma per le attrezzature di sollevamento dell'acqua (fig. 15: $\mathrm{S}, \mathrm{Da}, \mathrm{P}$ ).

La tipologia dell'impianto del calidarium e delle strutture relative è riconducibile, come le 'Terme della Regio VII', all'età tardo-antoniniana. Tuttavia sfuggono all'indagine quei settori dei bagni freddi, ad ovest, e della palestra, a sud, che furono riutilizzati nel complesso assai articolato della Basilica 3 e dei suoi annessi.

L'usanza di impiantare edifici di culto sopra o all'interno di strutture anche termali preesistenti è una caratteristica delle città dell'Africa romana. A Sabratha le difficili operazioni di riuso dovettero scattare negli anni immediatamente posteriori al terremoto del 365 d.C. e durarono fino all'invasione vandalica del 455, con successivi rifacimenti prebizantini, tra la fine del V ed i primi anni del VI secolo d.C.

\footnotetext{
${ }^{25}$ Bonacasa Carra 2012.

${ }^{26}$ Bonacasa Carra 2003-2004: 15-20, 55-57, figg. 22-23.

${ }^{27}$ Il nome 'Terme del Teatro' è stato attribuito - prima arbitrariamente dagli scavatori ed in seguito per comodità da noi editori - all'interessante ma assai più modesto edificio termale della Regio $\mathrm{V}$, di cui abbiamo appena detto.
} 


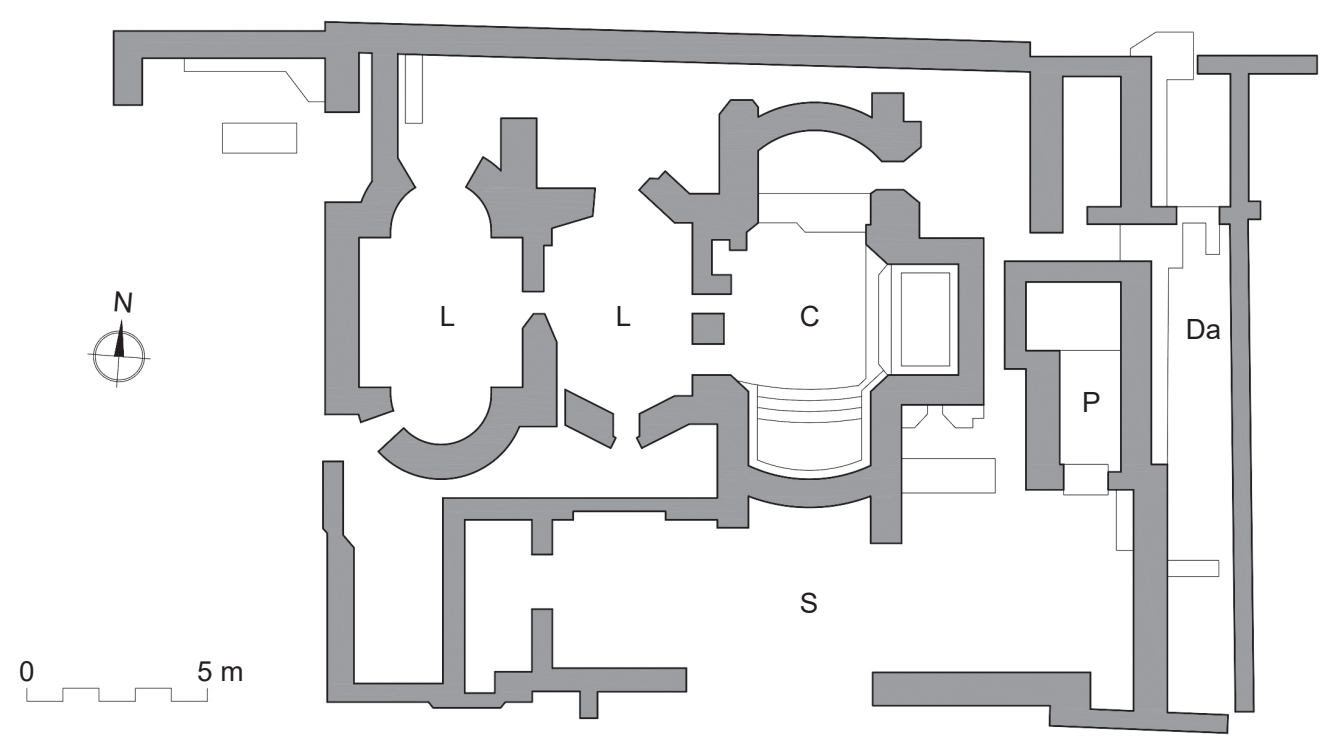

15. Pianta delle 'Terme sotto la basilica 3' (Disegno: F. Scirè (C) Archivo CeRAM, Università di Palermo).

\section{'TERME A MARE'}

Le 'Terme della Regio II', insula 9 furono chiamate dagli scavatori 'Terme a mare' per la posizione delle sale dei bagni rivolta verso la linea di costa (fig. 1), ma furono definite anche 'Terme della Ninfa' per la statua femminile acefala, a grandezza naturale (fig. 17), quasi certamente collocata nella nicchia che sovrastava a nord la vasca superstite del frigidarium (fig. 16). La scultura fu rinvenuta, sembra, in associazione con il torso di una statua maschile pure acefala ${ }^{28}$. Nonostante si tratti dell'unico edificio termale pubblico presente nella Regio II, certamente il secondo per estensione tra quelli noti a Sabratha, non ha ricevuto particolari attenzioni da parte degli editori. Riportato alla luce tra la fine del 1929 e il settembre del 1930 ha costituito sempre un problema per il cattivo stato di conservazione delle strutture, dovuto agli effetti dell'erosione marina.

\footnotetext{
${ }^{28}$ La relazione di scavo del 13-19 luglio 1930 così recita: Ad Ovest in direzione dell'ipotetica piscina, sul piano del pavimento si rinvengono due statue di marmo pario (?), una di Venere e l'altra di Apollo. Di fatto risulta una figura di Dioniso stante sulla gamba destra; la sinistra è leggermente arretrata e puntellata da un sostegno con l'attributo della vite col grappolo d'uva; sulle spalle (la destra leggermente sollevata rispetto alla sinistra) ricadono in lunghe ciocche sinuose i capelli; il busto dalle masse muscolari appena accennate asseconda con lo spostamento dell'anca il movimento della gamba destra. Potrebbe essere una copia romana del Dioniso tipo Woburn Abbey, attribuito a Leochares: Gasparri 1986: C, 413-514, 435, n. 120. Vedi anche la statua di Dioniso in marmo lunense nella Galleria Borghese (sala IV, inv. n. CXXXIV, alt. m 1.57) del 120-130 d.C. circa, dove la posa delle gambe è invertita. Per la provenienza delle due statue dalle 'Terme a Mare' vedi anche Ward 1999: 55. Un'altra statua di Dioniso rinvenuta a Sabratha nel frigidarium delle 'Terme della Regio VII' è stata attribuita all'ultimo quarto del II secolo d.C.: Brecciaroli Taborelli 1974-1975: 132, n. 52.
} 

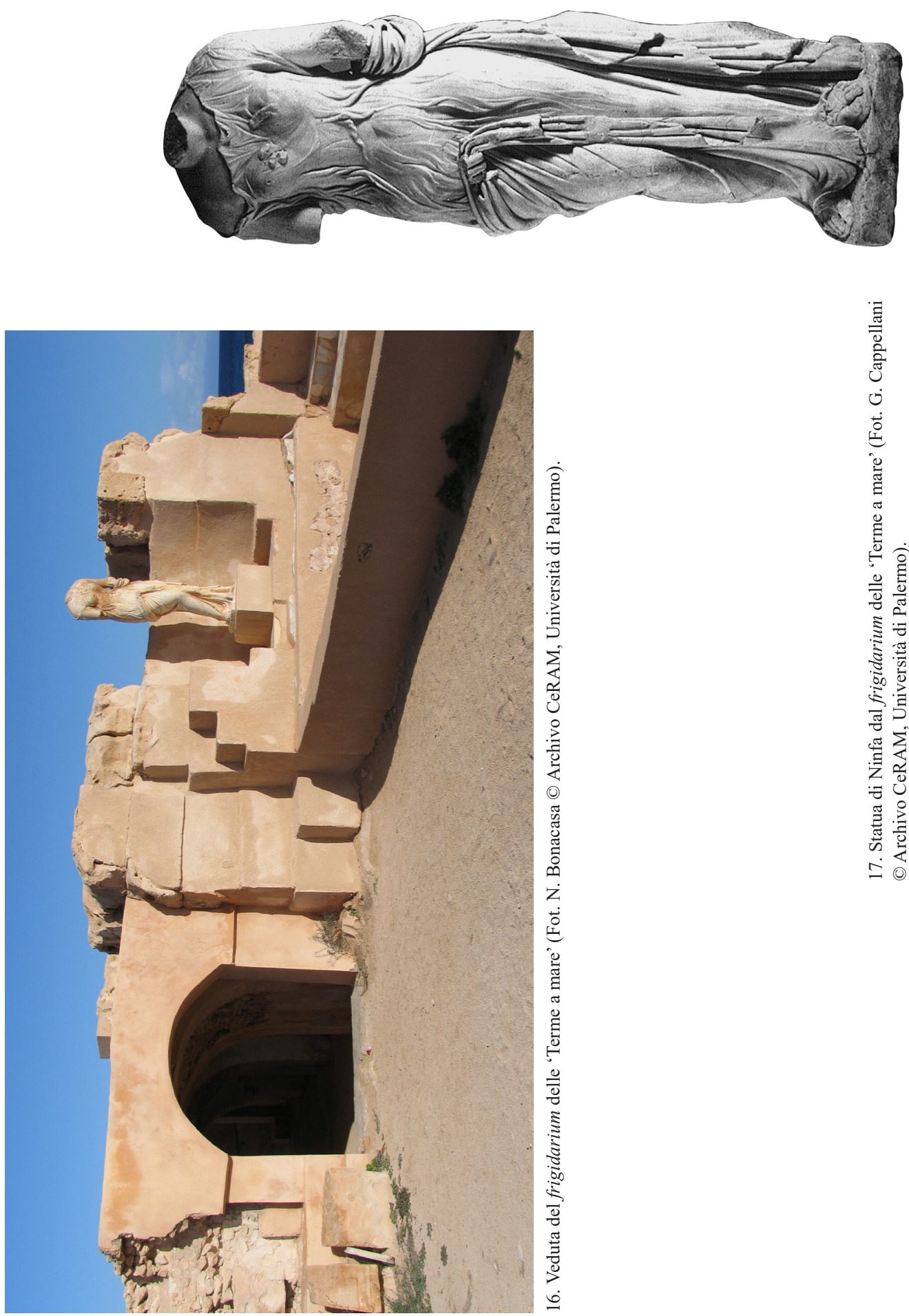
La mancanza di dati di scavo certi ci ha indotti a impegnarci soprattutto sull'analisi delle strutture superstiti - ricorrendo sia al rilievo archeologico sia al rilievo fotogrammetrico dell'intero fronte orientale, lungo la linea di costa, dove si estende il crollo delle murature delle sale dei bagni (fig. 18) - al fine di proporre un'ipotesi sostenibile di integrazione delle strutture oggi mancanti (fig. 19).

I dati topografici dimostrano che le 'Terme a mare' rappresentano un arbitrio urbanistico, in quanto furono inserite a forza tra l'angolo nord-est del portico del 'Tempio Orientale' (di Liber Pater), che concludeva ad est la piazza del foro, e la linea di costa (che in antico era più avanzata ${ }^{29}$ ), sovrapponendosi parzialmente alla maglia dei quartieri punici e proto-imperiali (fig. 1). L'impianto strutturale va certamente collocato dopo il terremoto di età flavia ${ }^{30} \mathrm{e}$, al più tardi, entro il primo trentennio del II secolo d.C. e rientrerebbe nel periodo successivo alla nuova sistemazione del Foro, con la basilica giudiziaria e la riedificazione del 'Tempio Orientale' ${ }^{31}$. L'area occupata misurava all'incirca 2,346 $\mathrm{mq}^{32}$.

L'edificio era imponente non tanto per l'estensione quanto per le solide strutture a blocchi isodomi realizzate lungo l'intero fronte sul mare, che formavano un sistema di muri portanti spesso in fondazione 3 metri circa; era caratterizzato da muri di spina, con andamento nord-est/sud-ovest, tra loro incatenati da muri trasversali, con andamento nord-ovest/sud-est - in modo da creare una struttura a telaio, a linea spezzata, che si estendeva al di sotto di ciascun ambiente dei bagni e che si legava a nord-ovest con un possente contrafforte aggettante a spigolo vivo su una base gradinata. Purtroppo, di questo sistema così complesso sopravvivono, oltre al contrafforte, alcuni paramenti murari percepibili all'interno di un esteso crollo a scarpa, che si dispone fino al limite estremo dell'attuale linea di costa (fig. 18).

La restituzione che noi proponiamo in pianta (fig. 19), nel rispetto dell'andamento che la linea di costa aveva in antico, prevede all'incirca il raddoppio della superficie di tutti gli ambienti prospicienti sul mare, con andamento a linea spezzata, fino a raggiungere il contrafforte a nord-est - punto di forza di tutto il versante orientale dell'edificio, che aveva alle spalle una monumentale e solida piattaforma studiata per il sollevamento, il deposito e la distribuzione corretta dell'acqua negli impianti. La nostra proposta di restituzione della pianta è sostenuta anche dallo studio metrologico, fondato sullo stesso modulo ' $\mathrm{M}$ ' utilizzato per le 'Terme della Regio V'33.

L'edificio della Regio II è caratterizzato da sette ambienti legati tra di loro, e dalla struttura indipendente della latrina. Tutti si affollano intorno ad un'area comune scoperta contenente: il pozzo, la piattaforma per il pompaggio dell'acqua e lo stoccaggio in apposite vasche, un

\footnotetext{
${ }^{29}$ Yorke 1986: 243-245, figg. 108-109.

${ }^{30}$ Che sarebbe accaduto tra il 64 e il 70 d.C. secondo Di Vita 2015: 650-653.

${ }^{31}$ Nel Periodo Ib, secondo Kenrick 1986: 312.

32 Bonacasa, Bonacasa Carra 2003: 417; Bonacasa 2010: 41.

${ }^{33}$ Bonacasa Carra 2012.
} 


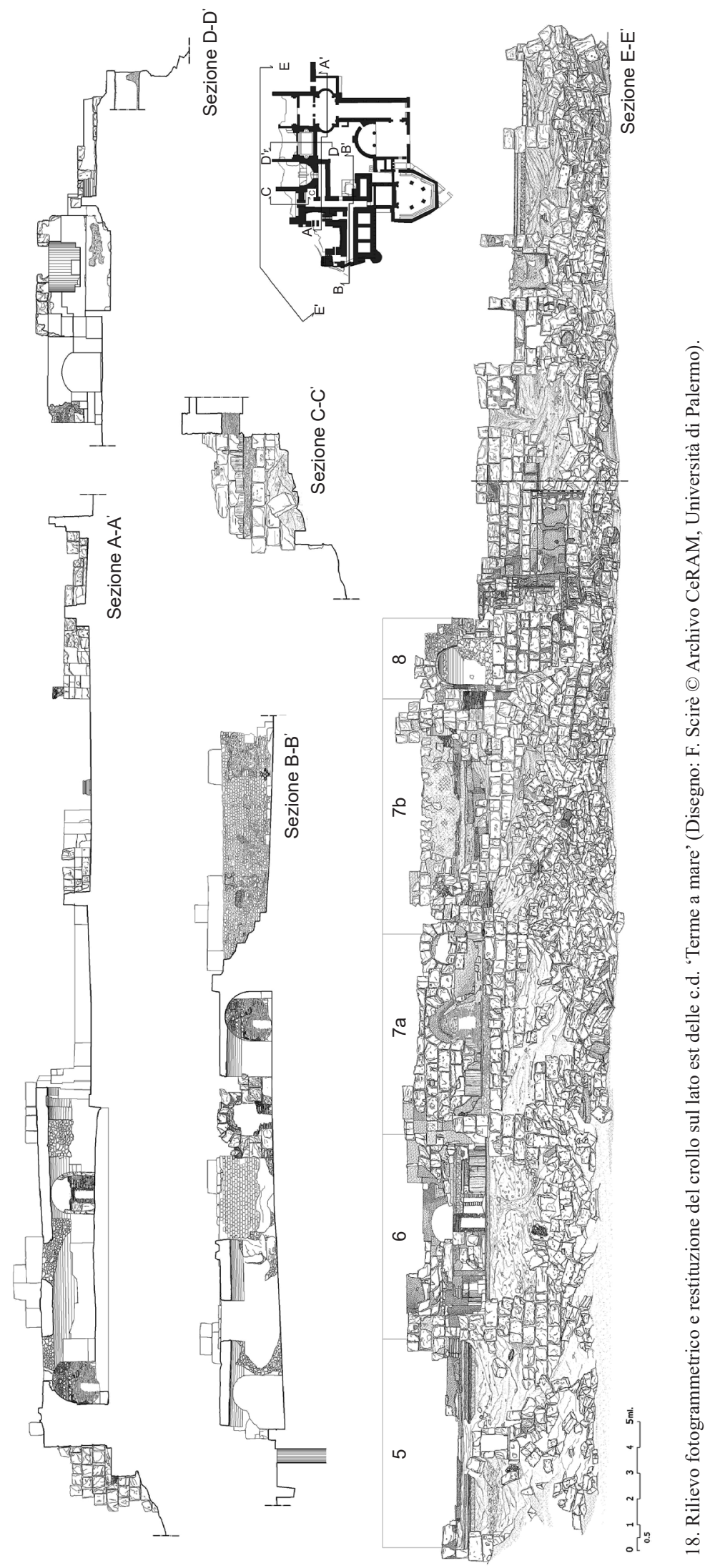


sistema di condotte per la distribuzione dell'acqua pura e una serie di canali fognari per lo smaltimento delle acque reflue.

Confortati dall'analisi dei frammenti di volte superstiti presenti nell'area di scavo ${ }^{34}$ siamo in grado di avanzare anche una ipotesi circa le coperture e quindi circa i volumi dell'edificio (fig. 20). Sulla fronte orientale, sia nelle sale dei bagni (fig. 19: 5, 6, 7b) che nei vani di disimpegno (fig. 19: 4, 7) si susseguivano quattro volte a crociera quasi identiche per dimensione, che ricoprivano la parte centrale di ciascun vano (fig. 20). Il figidarium oltre alla crociera (pari a $2 \times 2 \mathrm{M}$ ), ad ovest aveva una grande volta a botte (pari a 1 × $5 \mathrm{M}$ ), che ricopriva sia la natatio che il vicino ambiente $3 \mathrm{a}$. La seconda vasca fredda, ad est, doveva avere invece una volta indipendente, probabilmente una botte più piccola (di 1 x $2 \mathrm{M}$ ). Volte a botte analoghe per dimensioni e per altezza, dovevano ricoprire anche le tre vasche del calidarium, e la nicchia che comunicava col tepidarium (fig. 19: 7). La quinta volta a crociera che ricopriva il vestibolo (fig. 19: 1) era in assoluto la più monumentale: misurava 3 x $3 \mathrm{M}$ in pianta e 4,5 $\mathrm{M}$ in altezza; era indipendente dall'abside. Le finestre che illuminavano l'ambiente dovevano essere collocate in corrispondenza dei $2 / 3$ dell'altezza totale, come avviene in tutti i casi con copertura a crociera. I volumi di questa crociera certamente spiccavano sul piazzale antistante l'ingresso, anche rispetto alle altre coperture a botte che si susseguivano nei vani di attesa (fig. 19: 2-3). L'abside era separata dalla sala da un architrave poggiante su sue colonne e due pilastri angolari con capitelli e paraste di marmo bianco; le colonne erano di cipollino, le basi attiche e i capitelli corinzi di marmo bianco. L'abside - la cui altezza era pari a $3 \mathrm{M}$ - era coperta da una calotta corrispondente a $1 \frac{1}{4}$ di cupola ed era più bassa di 1,5 M rispetto allo sviluppo della crociera (figg. 19-20).

Il percorso di fruizione dei bagnanti (fig. 19) iniziava proprio dalla sala absidata, che si apriva sul piazzale triangolare con un portone preceduto da due colonne, le cui basi erano incassate nel lastricato dello stesso piazzale ${ }^{35}$. Questa sala, monumentale per lo sviluppo architettonico e la decorazione marmorea, fungeva da vestibolo agli spazi di attesa: il lungo e stretto corridoio ed il vano bipartito (fig. 19: 2-3) - entrambi dotati di panchine alle pareti. Una volta concluso il periodo di attesa, ciascun fruitore poteva transitare dallo spogliatoio adiacente al frigidarium e da quest'ultimo accedere direttamente al settore dei bagni caldi secondo la sequenza: sudatio (laconicum?) - tepidarium - calidarium (fig. 19: 6-7). Le operazioni del bagno si concludevano nel frigidarium e con un percorso retrogrado 'a doppio senso' si guadagnava l'uscita - attraversando di nuovo il vestibolo ${ }^{36}$.

\footnotetext{
${ }^{34}$ Sono presenti nell'area di scavo, all'esterno dell'edificio, undici frammenti architettonici - alcuni dei quali sono stati rilevati per confermare le ipotesi avanzate per la copertura dei vani. Si tratta di un frammento di forma triangolare rivestito di intonaco con 3 superfici rilevabili, pertinente all'arco di passaggio tra i vani 2 e 3 . Un altro grosso frammento era pertinente alla volta del corridoio 2: consiste in una parte angolare strutturale in mattoni di cotto e lastrine di arenaria, queste ultime si trovavano all'imposta tra pilastro e arco; le file di mattoni erano lo spicco dell'arco, gli uni e le altre erano legati con strati di malta e pozzolana. Altri tre frammenti triangolari, con mattoni di cotto appartenevano probabilmente alla grande crociera della sala absidata 1 .

${ }^{35}$ È possibile che le colonne reggessero un timpano (fig. 20).

${ }^{36}$ Bonacasa Carra 2012; Bonacasa Carra, Scirè 2012.
} 


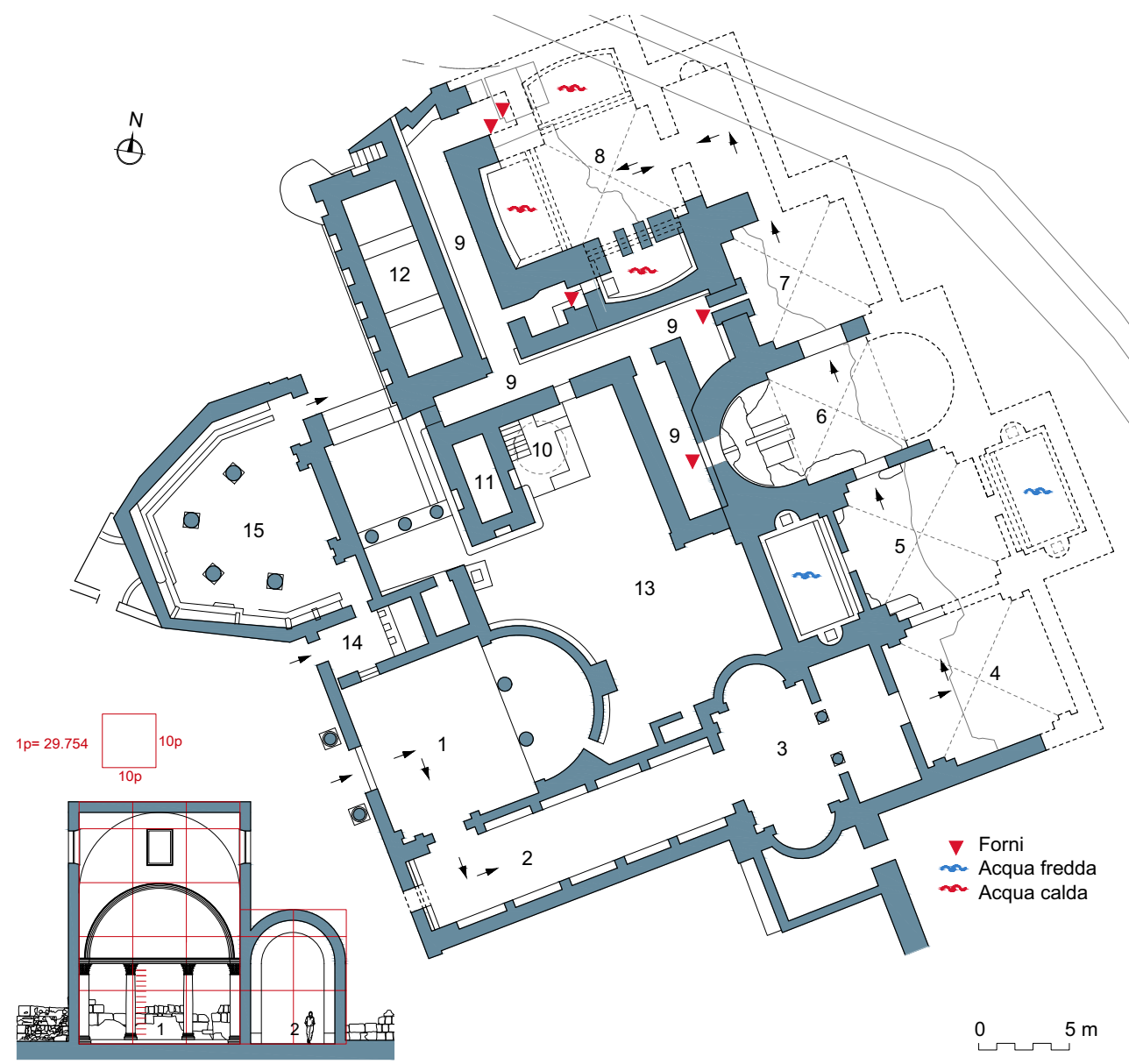

19. Pianta delle c.d. 'Terme a mare' nella Regio II: 1. salone d'ingresso; 2. corridoio; 3. spogliatoio; 4. anticamera; 5. frigidarium; 6. tepidarium; 7. laconicum; 8. calidarium; 9. corridoio di servizio; 10. pozzo; 11. piattaforma di sollevamento acqua; 12. cisterne; 13. ambiente di servizio scoperto; 14. ingresso latrina; 15. latrina (Disegno: F. Scirè (C) Archivo CeRAM, Università di Palermo).

Il cedimento del versante orientale dell'edificio (fig. 18) - per motivi da attribuire ragionevolmente ad un evento catastrofico, di cui ci sfuggono le dinamiche - avrebbe determinato la distruzione parziale del settore dei bagni caldi. Il rinvenimento, sullo spigolo nord-est della vasca meridionale, di un AE4 di età costantiniana ci conforta nell'ipotesi che anche queste terme abbiano potuto risentire gli effetti delle vicende che sconvolsero Sabratha nel IV secolo d.C. La presenza di un muro a gradoni con sviluppo curvilineo in direzione est-ovest che si appoggia al contrafforte con base gradinata legandolo alle strutture del tepidarium, lascia supporre che l'ambiente di ingresso e la vasca nord del calidarium fossero irrimediabilmente perduti, mentre le altre due vasche avrebbero continuato a funzionare almeno per un certo periodo. 


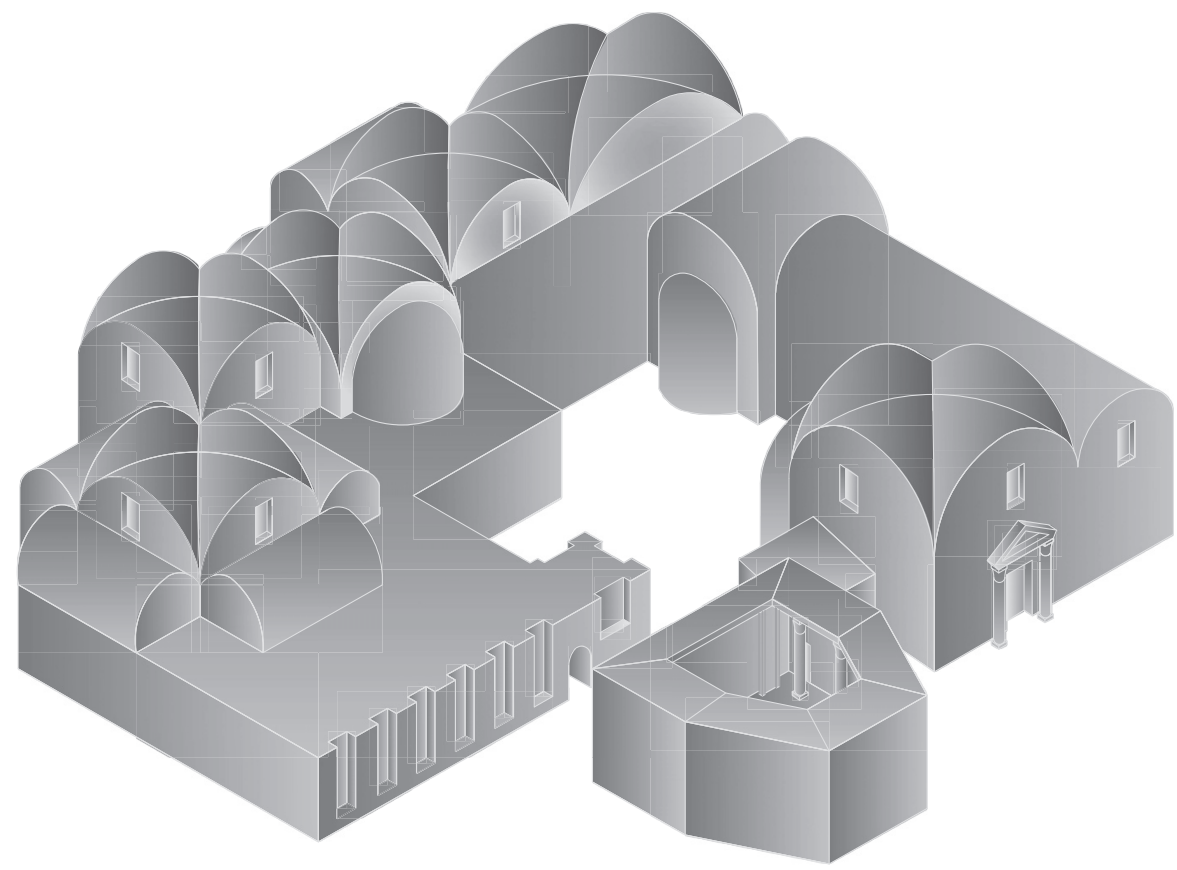

b

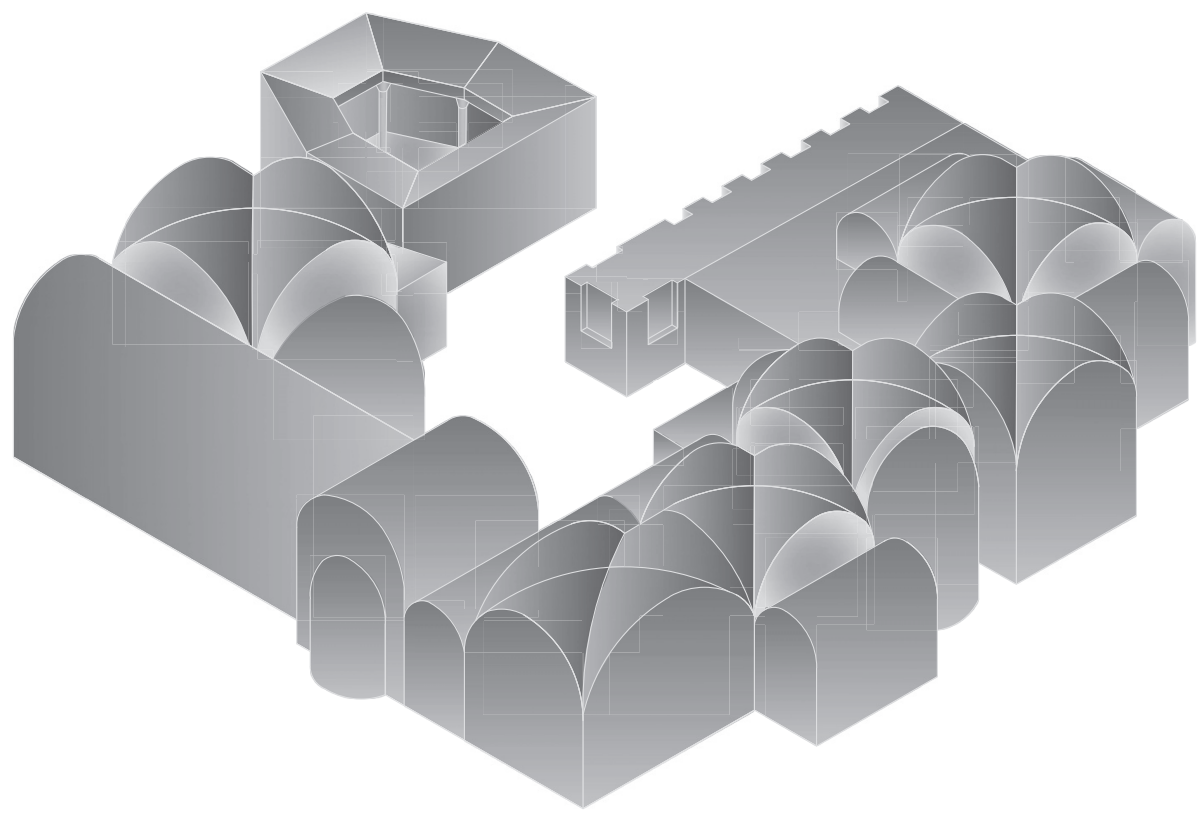

20. Ipotesi di restituzione dei volumi delle c.d. 'Terme a mare': a. vista dal ovest; b. vista dal sud (Disegno: F. Scirè (C) Archivo CeRAM, Università di Palermo). 
Lo stato di conservazione delle strutture - a differenza di quanto si è potuto riscontrare nelle 'Terme della Regio V' - non ci consente di ipotizzare per le 'Terme a mare' né ampliamenti, né modifiche dal punto di vista strutturale. Siamo certi solo di alcune opere di manutenzione ai pavimenti ed alle pareti degli ambienti più conservati perché meno esposti agli effetti dell'erosione marina. Sicché la struttura progettata e realizzata nel II secolo d.C. riteniamo che sia stata efficiente e funzionante in tutte le sue parti fino al IV - quando venne coinvolta nella serie di eventi catastrofici che sconvolsero la città di Sabratha. Ciò nonostante fu messa nelle condizioni di sicurezza che consentissero di mantenere la sua fruizione di impianto termale e forse anche la rifunzionalizzazione del vestibolo absidato come aula di culto cristiano nell'avanzato VI secolo.

Come ha sostenuto di recente Nicola Bonacasa, nessun edificio termale di Sabratha è comparabile con la grandiosità funzionale delle 'Terme adrianèe' di Leptis Magna, né con la raffinatezza delle piccole 'Terme della caccia' della stessa Leptis ${ }^{37}$. Eppure in Tripolitania si percepisce bene che agli inizi del II secolo d.C. le tipologie generali e le componenti interne dell'impianto termale romano erano già state fissate, individuando nel frigidarium il fulcro dell'intera struttura. Le planimetrie degli edifici sabrathensi che abbiamo appena illustrato appaiono altamente concentrate e funzionali nella loro disposizione assiale. I frigidaria sono ampi e serviti da apodyteria, e soprattutto dotati di ampi luoghi di attesa e di attrezzate latrine - insomma, spazi bene organizzati e accessibili facilmente dai frequentatori, oltre che dotati di percorsi adeguati e bene auguranti come dimostrano i mosaici delle 'Terme del Teatro'. Frigidario e latrina erano nelle 'Terme a mare' gli spazi privilegiati per l'inserimento di arredi scultorei: statue di Afrodite/Ninfa, di Dioniso, di una Afrodite pudica e una statuetta di Abbondanza di fattura mediocre ${ }^{38}$.

Gli spazi destinati al bagno caldo in tutti gli edifici sabrathensi si distinguono per la sequenza e la compattezza dei locali specializzati (laconicum, tepidarium) e per il modello del calidarium bene orientato con vasche indipendenti rispetto alla sala nella struttura delle volte e nella localizzazione e organizzazione dei praefurnia, sempre curati in tutti gli impianti. Le 'Terme a mare' spiccano proprio per la grandiosità dei corridoi di servizio - concepiti ad altezza d'uomo per favorire il funzionamento capillare, ma autonomo, di ogni spazio riscaldato.

In conclusione ci sentiamo di poter affermare che l'architettura termale a Sabratha si sviluppa nel corso del II secolo d.C. - sia nella riorganizzazione degli spazi del foro dopo il terremoto di età flavia, sia nella pianificazione del nuovi quartieri di espansione intorno al teatro e a sud ed ovest del foro nelle aree della Regio VI e della Regio VII. In queste nuove realtà urbanistiche si impongono le 'Terme a mare' per le originali intuizioni tecnico-strutturali - finalizzate a disporre di un'area edificabile maggiore di quanto potesse offrire la tettonica del suolo. Ma si impongono anche le strutture ben conservate

\footnotetext{
${ }^{37}$ Bianchi Bandinelli, Vergara Caffarelli, Caputo 1963: 99-100, 105-106; Yegül 1992: 186-192, $242-243$.

${ }^{38}$ Bonacasa 2010: 42-48.
} 
delle 'Terme del Teatro', che hanno favorito la nostra sperimentazione di nuove tecniche di analisi finalizzate alla restituzione del progetto originario.

\section{Riferimenti}

Aiosa, S. 2012: Urbanistica e ideologia: a proposito del Tempio di Ercole a Sabratha, [in:] Cocco, M.B., Gavini, A., Ibba, A. (a cura di), L'Africa Romana. Trasformazione dei paesaggi del potere nell'Africa settentrionale fino alla fine del mondo antico. Atti del XIX convegno di studio, Sassari 16-19 dicembre 2010, I, Roma, 311-324

Aiosa, S. 2013: Il tempio di Ercole a Sabratha. Architettura e contesto urbano, $M A L$ XXXIII, Roma

Barone, G. 1994: Gessi del Museo di Sabratha, MAL XXXIII, Roma

Bianchi Bandinelli, R., Vergara Caffarelli, E., Caputo, G. 1963: Leptis Magna, Roma

Bonacasa, N. 1960: Segnalazioni Alessandrine II. Sculture minori del Museo Greco Romano di Alessandria, ArchClass XII, 170-188

Bonacasa, N. 2004: Sabratha. Terme di Oceano, restauro dei mosaici 1999-2002, [in:] Meli, G. (a cura di), Apparati musivi antichi nell'area del Mediterraneo. Conservazione programmata e recupero. Contributi analitici alla carta del rischio: Atti del I Convegno internazionale di studi "La materia e i segni della storia", Piazza Armerina, 9-13 aprile 2003, Palermo, 328-331

Bonacasa, N. 2010: Roma in area punica. Le terme di Sabratha, [in:] Incontri tra culture nel mondo mediterraneo antico. Atti del XVII Congresso Internazionale di Archeologia Classica (Roma 22-26 settembre 2008), Bollettino di archeologia on line I (volume speciale Congresso A.I.A.C. 2008), 37-54, http://www.bollettinodiarcheologiaonline.beniculturali.it/ (accesso: 6 aprile 2016)

Bonacasa, N., Bonacasa Carra, R.M. 2003: Gli edifici termali di Sabratha. Nota preliminare, $Q A L 18,403-419$

Bonacasa Carra, R.M. 2012: Sabratha. Le trasformazioni dello spazio urbano e lo sviluppo delle aree paleocristiane nelle Regiones III-V: studio preliminare per un GIS archeologico, [in:] Bonacasa Carra, R.M. (a cura di), Pagani e cristiani a Sabratha e Leptis Magna tra III e VI secolo d.C. Monumenti e reperti, tradizione e immagini, Atti del seminario di studio, Polo Universitario della Provincia di Agrigento 26-27 gennaio 2012, Palermo, 47-76

Bonacasa Carra, R.M. 2016: Gli edifici termali della Regio II e della Regio V di Sabratha, dal progetto alla fruizione, [in:] Bonacasa, N. (a cura di), Sabratha. Atti dei Convegni Lincei 299. Giornate di studio: L'archeologia del sacro e l'archeologia del culto, Roma 8-11 ottobre 2013, Roma, 189-260

Bonacasa Carra, R.M., Morfino, D. 2003-2004: Il cristianesimo a Sabratha alla luce delle più recenti indagini, RAR LXXVI, 3-77

Bonacasa Carra, R.M., Scirè, F. 2012: Sabratha: le fasi dell'edificio termale a NO del Teatro attraverso l'analisi delle strutture, [in:] Cocco, M.B., Gavini, A., Ibba, A. (a cura di), L'Africa Romana. Trasformazione dei paesaggi del potere nell'Africa 
settentrionale fino alla fine del mondo antico. Atti del XIX convegno di studio, Sassari 16-19 dicembre 2010, I, Roma, 365-382

Brecciaroli Taborelli, L. 1974-1975: Le terme della Regio VII a Sabratha, LibAnt XI-XII, 113-146

Caputo, G., Ghedini, F. 1984: Il tempio d'Ercole di Sabratha, MAL XIX, Roma

Cipriano, G. 2012: Sabratha. Distribuzione topografica e consistenza delle aree funerarie adiacenti le basiliche cristiane a Nord del Teatro: dalla cosiddetta "area ad L" alle Terme di Oceano, [in:] Bonacasa Carra, R.M. (a cura di), Pagani e Cristiani a Sabratha e Leptis Magna tra III e VI secolo d. C. Monumenti e reperti, tradizione e immagini: Atti del seminario di studio, Polo universitario della Provincia di Agrigento 26-27 Gennaio 2012, Palermo, 101-130

Di Vita, A. 1980: Evidenza dei Terremoti del 306-310 e del 365 d.C. in Tunisia, Antiquités africaines 15, 303-307

Di Vita, A. 1990: Sismi, urbanistica e cronologia assoluta. Terremoti e urbanistica nelle città di Tripolitania fra il I secolo a.C. ed il IV d.C. Actes du colloque, École française de Rome 3-5 décembre 1987, Paris-Rome, 425-494

Di Vita A. 2015: Scritti Africani, MAL XXXVIII, a cura di M.A. Rizzo Di Vita, G. Di Vita Evrard, Roma

Di Vita, A., Procaccini, P., Pucci, G. 1974-1975: Lo scavo a Nord del mausoleo punico-ellenistico A di Sabratha, LibAnt XI-XII, 7-22

Floriani Squarciapino, M. 1941: Un gruppo di Orfeo tra le fiere del Museo di Sabratha, Bullettino del Museo dell'Impero Romano 12, Appendice a Bullettino della Commissione archeologica comunale di Roma LXIX, 61-79

Gasparri, C. 1986: Dionysos, [in:] LIMC III/1, 414-514

Kenrick, Ph.M. 1986: Excavations at Sabratha 1948-1951, JRS Monograph 2, London

Macaluso, R. 1995: Un tesoretto di denari di età severiana da Sabratha, $Q A L 17$, 75-81

Mandruzzato, A. 2012: Edilizia privata a Sabratha. Considerazioni preliminari sulla Casa del Peristilio (Regio V, ins. 2), [in:] Bonacasa Carra, R.M. (a cura di), Pagani e cristiani a Sabratha e Leptis Magna tra III e VI secolo d.C. Monumenti e reperti, tradizione e immagini, Atti del seminario di studio, Polo Universitario della Provincia di Agrigento 26-27 gennaio 2012, Palermo, 31-46

Nielsen, I. 1990: Thermae et Balnea. The Architecture and Cultural History of Roman Public Baths, Aarhus

Pasquinucci, M. (a cura di) 1987: Terme romane e vita quotidiana, Modena

Pesce, G. 1953: Il tempio d'Iside in Sabratha, MAL IV, Roma

Stucchi, S. 1957: Le piante delle terme romane d'Africa ed i loro rapporti con quelle di Roma e dell'Europa, [in:] Atti del V Convegno nazionale di storia dell'architettura. Perugia, 23 settembre 1948, Firenze, 177-186

Thébert, Y. 1991: Problèmes de circulation dans les thermes d'Afrique du Nord, [in:] Les thermes romains. Actes de la table ronde, Rome, 11-12 novembre 1988, CEFR 142, Rome, 139-149 
Thébert, Y. 2003: Thermes romains d'Afrique du Nord et leur contexte méditerranéen études d'histoire et d'archéologie, BEFAR 315, Rome

Yegül, F. 1992: Baths and Bathing in Classical Antiquity, New York-Cambridge, Mass.

Yorke, R.A. 1986: The harbour, [in:] Kenrick, Ph.M. (a cura di), Excavations at Sabratha 1948-1951, JRS Monograph 2, London, 242-245

Warot, S. 1960: Timgad: bene lava, Libyca VIII/2, 167-172

Ward, Ph. 1970: Sabratha. A Guide for Visitors, London 


\section{ÉTUDES et TRAVAUX XXX / 2017}

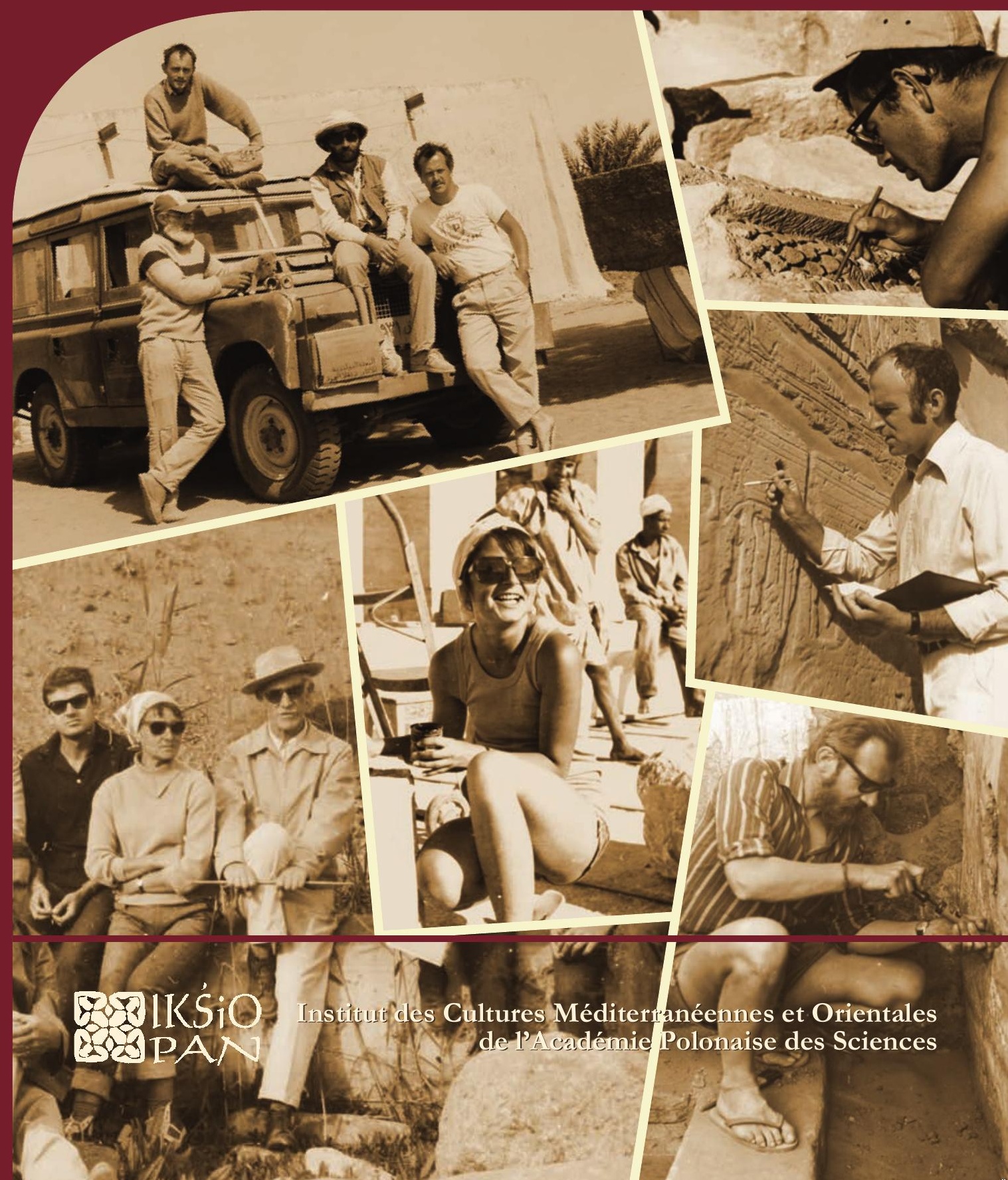




\title{
COMITÉ DE RÉDACTION SCIENTIFIQUE
}

Maciej Makowski - rédacteur en chef

Jadwiga Iwaszczuk - rédacteur et sécretaire de la rédaction

Mariusz Drzewiecki - rédacteur

Maciej G. Witkowski - rédacteur

\section{CONSEIL SCIENTIFIQUE DU JOURNAL}

M. Kobusiewicz (IAE PAS, Warszawa), E. Laskowska-Kusztal (IMOC PAS, Warszawa),

D. Michaelides (University of Cyprus, Nicosia),

J.Ch. Moretti (IRAA-MOM, Université de Lyon 2/CNRS),

D. Raue (Ägyptisches Museum der Universität Leipzig), P. Reynolds (ICREA, Barcelona),

D. Welsby (British Museum, London)

\section{COMITÉ SCIENTIFIQUE DE LECTURE}

J. Holaubek (Institut für Ägyptologie, Wien), S. Ikram (AUC, Cairo),

K. Innemée (Universiteit Leiden), J. McKenzie (Faculty of Oriental Studies, University of Oxford),

N. Strudwick (University of Cambridge), A. Loprieno-Gnirs (Universität Basel),

Ch.E. Loeben (Museen für Kulturgeschichte, Hannover), Y. Tristant (Macquarie University, Sydney),

V.W.J. van Gerven Oei (University of Aberdeen), A. Peignard-Giros (HiSoMA-MOM, Université de Lyon 2/CNRS), J.A. Ostrowski, E. Papuci-Władyka, J. Śliwa (IA JU, Kraków), R. Czerner (WUST, Wrocław), A. Ćwiek (IA AMU, Poznań), M. Wiewióra (IA NCU, Toruń), K. Domżalski

(IAE PAS, Warszawa), K.O. Kuraszkiewicz (DE FOS UW), M. Barwik, P. Bieliński, P. Dyczek, W. Godlewski, D. Ławecka, S. Rzepka, J. Żelazowski, M. Gawlikowski, J. Młynarczyk, A. Niwiński, T. Sarnowski, D. Szeląg, T. Waliszewski (IA UW, Warszawa)

\section{RÉDACTEUR THÉMATIQUE DU VOLUME \\ Barbara Lichocka}

\author{
AIDE RÉDACTION TECHNIQUE \\ Dorota Dobrzyńska, Mariusz Drzewiecki
}

REVUE DES TEXTES ANGLAIS

Jo Harper 
ÉTUDES et TRAVAUX XXX 
INSTYTUT KULTUR ŚRÓDZIEMNOMORSKICH I ORIENTALNYCH POLSKIEJ AKADEMII NAUK

\section{STUDIA i PRACE}

XXX

\section{Ro IKŚSiO \\ ESA PAN}

WARSZAWA

2017 
INSTITUT DES CULTURES MÉDITERRANÉENNES ET ORIENTALES DE L'ACADÉMIE POLONAISE DES SCIENCES

\section{ÉTUDES et TRAVAUX}

XXX

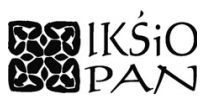

VARSOVIE

2017 
Publication scientifique financée dans le cadre du programme du Ministre de la Science et de l'Éducation Supérieure

« Programme National de Développement de l’Humanistique » pour les années 2016-2021 (projet no 3bH 150099 83)

\title{
HARODOWY PROGRAM ROZWOJU HUMANISTYKI
}

\author{
Copyright (C) \\ Instytut Kultur Śródziemnomorskich i Orientalnych PAN \\ et les Auteurs \\ Warszawa 2017
}

\author{
ISSN 2084-6762 \\ (avant $2011: 0079-3566$ ) \\ e-ISSN 2449-9579 \\ Version première en papier, imprimée en Pologne - 150 copies \\ Version électronique accessible sur \\ http://www.etudesettravaux.iksiopan.pl
}

Édition: Polskie Towarzystwo Historyczne et Wydawnictwo Neriton, Warszawa

Conception générale de couverture : J. Iwaszczuk

Photos de couverture : En haut, à gauche. Vieille Dongola 1991, S. Jakobielski

(debout à gauche), K. Pluskota (debout à droite), B. Żurawski (assis sur le camion)

et P. Wierzbicki (assis sur le camion) (de la collection de B. Żurawski)

En haut, à droite. Palmyre 1964, M. Marciniak au travail (phot. A. Dziewanowski)

Au centre. E. Laskowska-Kusztal au travail (de la collection de E. Laskowska-Kusztal)

En bas, à gauche. Tell Atrib 1962 ; de gauche : T. Biniewski, M. Marciniak, K. Kołodziejczyk,

K. Michałowski, A. Ostrasz, S. Jakobielski et S. Jasiewicz devant eux

(de la collection de IKŚSiO PAN).

En bas, à droite. Vieille Dongola 1976, S. Jakobielski nettoyant le mur (phot. M. Steinborn).

Au centre, à droite, K. Myśliwiec en train des travaux de documentation (de la collection de IKŚiO PAN) 


\section{Table des matières}

BARBARA LICHOCKA

Ergon agathon

Hartwig Altenmüller

$\mathrm{Zu}$ den Feindbildern auf den Zauberstäben des Mittleren Reiches und der Zweiten

Zwischenzeit

Nathalie Beaux

Des $m s w n s w$ de Thoutmosis III à Deir el-Bahari

Briant Bohleke, Nigel Strudwick

A Label for Opening of the Mouth Implements from the Burial of Senneferi (TT99)

and Remarks on the Ritual

Rosa Maria Bonacasa Carra, Nicola Bonacasa

Nuovi dati sugli edifici termali di Sabratha

EDWARD BROVARSKI

A Fragmentary Carrying Chair Scene in Salt Lake City, Utah

Julia Burdajewicz

Wall Painting Decoration from the North-West Church in Hippos-Sussita

of the Decapolis

Mariusz BURDAJEWICZ

From Pagan Temple to Church in Late Antiquity Palestine. A View from

Hippos-Sussita

MAREK ChlodNicki

Early Dynastic Bead Workshops at the Central Kom of Tell el-Farkha.

Patryk ChudziK, Mariusz Caban

Observations on the Architecture of the Tomb of Horhotep in Western Thebes

Krzysztof M. Cialowicz

New Discoveries at Tell el-Farkha and the Beginnings of the Egyptian State.

Amr EL-TiebI

Four Wooden New Kingdom Female Statuettes in the Egyptian Museum, Cairo 


\section{Naguib KanaWATI}

Ritual Marriage Alliances and Consolidation of Power in Middle Egypt during the Middle Kingdom

Adam Łajtar, Jolanta Mlynarczyk

A Faction Acclamation Incised on a Pithos Found Near the North-West Church at Hippos (Sussita)

Adam ŁaJTAR, Grzegorz OchaŁa

Two Private Prayers in Wall Inscriptions in the Faras Cathedral

Adam Łajtar, Anna Poludnikiewicz

Medicinal Vessels from Tell Atrib (Egypt)

JaCeK Michniewicz, Jolanta MlynarczyK

Petrographic Variability of the Fabrics of Wine Jars from Sha'ar-Ha Amakim as a Reflection of Differences in Their Provenance and Chronology

Iwona ModrzewsKa-PianetTI

Les importations d'amphores Dressel 20 en Gaule Cisalpine

Arthur SEgal

Samaria-Sebaste. Portrait of a polis in the Heart of Samaria 409

JOACHIM ŚLIWA

The Motif of a 'Blind Harper' in an Unexpected Place

MONIKA WIĘCH

Searching for the Kitchen in the Early Roman Phase of the 'Hellenistic' House at Nea Paphos (Cyprus)

Abréviations 
THE VOLUME IS PUBLISHED TO CELEBRATE

THE $60^{\text {TH }}$ ANNIVERSARY

OF THE ESTABLISHMENT OF

THE RESEARCH CENTRE FOR MEDITERRANEAN ARCHAEOLOGY POLISH ACADEMY OF SCIENCES

FOUNDED IN 1956

WHOSE MISSION IS CONTINUED BY

THE INSTITUTE OF MEDITERRANEAN AND ORIENTAL CULTURES

OF THE POLISH ACADEMY OF SCIENCES 